\title{
Ordered Rate Constitutive Theories for Non-Classical Thermoviscoelastic Fluids with Internal Rotation Rates
}

\author{
K. S. Surana ${ }^{1}$, S. W. Long ${ }^{1}$, J. N. Reddy ${ }^{2}$ \\ ${ }^{1}$ Department of Mechanical Engineering, University of Kansas, Lawrence, KS, USA \\ ${ }^{2}$ Department of Mechanical Engineering, Texas A \& M University, College Station, TX, USA \\ Email: kssurana@ku.edu
}

How to cite this paper: Surana, K.S., Long, S.W. and Reddy, J.N. (2018) Ordered Rate Constitutive Theories for Non-Classical Thermoviscoelastic Fluids with Internal Rotation Rates. Applied Mathematics, 9, 907-939. https://doi.org/10.4236/am.2018.98063

Received: June 21, 2018

Accepted: August 19, 2018

Published: August 22, 2018

Copyright $\odot 2018$ by authors and Scientific Research Publishing Inc. This work is licensed under the Creative Commons Attribution International License (CC BY 4.0).

http://creativecommons.org/licenses/by/4.0/

(c) (i) Open Access

\begin{abstract}
The paper presents constitutive theories for non-classical thermoviscoelastic fluids with dissipation and memory using a thermodynamic framework based on entirety of velocity gradient tensor. Thus, the conservation and the balance laws used in this work incorporate symmetric as well as antisymmetric part of the velocity gradient tensor. The constitutive theories derived here hold in coand contra-variant bases as well as in Jaumann rates and are derived using convected time derivatives of Green's and Almansi strain tensors as well as the Cauchy stress tensor and its convected time derivatives in appropriate bases. The constitutive theories are presented in the absence as well as in the presence of the balance of moment of moments as balance law. It is shown that the dissipation mechanism and the fading memory in such fluids are due to stress rates as well as moment rates and their conjugates. The material coefficients are derived for the general forms of the constitutive theories based on integrity. Simplified linear (or quasi-linear) forms of the constitutive theories are also presented. Maxwell, Oldroyd-B and Giesekus constitutive models for non-classical thermoviscoelastic fluids are derived and are compared with those derived based on classical continuum mechanics. Both, compressible and incompressible thermoviscoelastic fluids are considered.
\end{abstract}

\section{Keywords}

Rate Constitutive Theories, Non-Classical Thermofluids, With Memory, Convected Time Derivatives, Internal Rotation Gradient Tensor, Generators and Invariants, Cauchy Moment Tensor

\section{Introduction}

The conservation and the balance laws used in the derivation of the ordered rate 
constitutive theories presented here for the thermoviscoelastic non-classical fluids have been derived and presented by Surana et al. [1] [2] [3] [4] for solid and fluent continua in Lagrangian as well as Eulerian descriptions. For the benefit of the readers and for the sake of completeness we briefly describe the motivation behind non-classical continuum theory incorporating internal rotation rates as well as a brief description of the development of the theory. In complex flows the velocity gradient tensor varies between a location and its neighboring locations. Polar decomposition of the velocity gradient tensor or its decomposition into symmetric and skew symmetric tensors shows that varying velocity gradient tensor results in varying rotation rates between the neighboring locations. Since these varying rotation rates arise due to varying deformation of the continua, hence are completely defined by the deformation (antisymmetric part of the velocity gradient tensor), thus do not require additional degrees of freedom at a material point. If the internally varying rotation rates are resisted by the deforming fluid, then there must exist corresponding conjugate moments. This physics is all internal to the deforming continua and is present in all flows but is completely neglected in the presently used classical continuum theories for fluent continua. The continuum theory presented in references [3] [4] for fluent continua considers internal varying rotation rates in addition to the strain rate tensor between the neighboring material points (or locations) and the associated conjugate moments in the derivation of the conservation and the balance laws. This theory has been referred to as "internal polar theory" or non-classical continuum theory with internal rotation rates.

There is much published work on non-classical continuum theories under the titles: micropolar theories, stress couple theories, rotation gradient theories, strain gradient theories with applications to beams, shells, plates, vibrations, etc. [5]-[37]. A comprehensive review of these works can be found in references [1] [2] [3] [4] and others [5]-[37]. This is not repeated here for the sake of brevity. In this paper we utilize the conservation and the balance laws presented in references [4] [5] to derive constitutive theories for thermoviscoelastic fluids, both compressible and incompressible. The ordered rate constitutive theories for compressible and incompressible thermoelastic solids, thermoviscoelastic solids with and without memory, thermofluids and thermoviscoelastic fluids within the framework of conservation and balance laws of classical mechanics have been presented by Surana et al. [38]-[46]. The ordered rate constitutive theories for non-classical solid and fluent continua in which internal rotations due to Jacobian of deformation and the internal rotation rates due to velocity gradient tensor are considered also have been presented by Surana et al. [47]-[53]. The concepts used in these works leading to ordered rate constitutive theories of various orders are utilized in the present work.

The constitutive theories in this paper are derived in a basis independent manner, hence are valid for co- and contra-variant bases as well as for Jaumann rates. Entropy inequality and other conservation and balance laws are used to determine the constitutive variables. Their argument tensors are decided based 
on the conjugate pairs appearing in the entropy inequality as well as the additional desired physics that is not obvious from the conjugate pairs in the entropy inequality. Argument tensors of the constitutive variables as well as the choice of the constitutive variables are generalized to include convected time derivatives of the stress and the strain tensors up to certain orders giving rise to the ordered rate constitutive theories. The constitutive theories are primarily derived using representation theorem [54]-[73], i.e., theory of generators and invariants. Material coefficients for each constitutive theory are established using Taylor series expansion of the coefficients in a linear combination about a known configuration. It is shown that Maxwell model, Oldroyd-B model and Giesekus constitutive models based on classical mechanics are a subset of the constitutive theories derived here. Furthermore, it is shown that single constitutive theories for stress tensor, moment tensor, and heat vector derived based on integrity based on the non-classical mechanics also degenerates to the Maxwell, Oldroyd-B and Giesekus models derived in classical continuum mechanics. That is only a single constitutive model derived here is sufficient to represent dilute and dense polymer physics for classical as well as non-classical cases.

\section{Notations}

In the following we give a brief explanation of the notations. This is necessary as some of the notations are new [38]. If $x_{i}$ and $\bar{x}_{i}$ denote the position coordinates of a material point in the reference and current configurations respectively in a fixed frame ( $x$-frame), then

$$
\begin{gathered}
\bar{x}_{i}=\bar{x}_{i}\left(x_{1}, x_{2}, x_{3}, t\right) \\
\text { or } \quad x_{i}=x_{i}\left(\bar{x}_{1}, \bar{x}_{2}, \bar{x}_{3}, t\right)
\end{gathered}
$$

If $\{d x\}=\left[d x_{1}, d x_{2}, d x_{3}\right]^{\mathrm{T}}$ and $\{d \bar{x}\}=\left[d \bar{x}_{1}, d \bar{x}_{2}, d \bar{x}_{3}\right]^{\mathrm{T}}$ are the components of length $d s$ and $d \bar{s}$ in the reference and current configurations, and if we neglect the infinitesimals of orders two and higher in both configurations, then we obtain,

$$
\begin{array}{r}
\{d \bar{x}\}=[J]\{d x\} \\
\{d x\}=[\bar{J}]\{d \bar{x}\}
\end{array}
$$

with

$$
[J]=[\bar{J}]^{-1} ; \quad[\bar{J}]=[J]^{-1} ; \quad[J][\bar{J}]=[\bar{J}][J]=[I]
$$

and using Murnaghan's notation [74]

$$
[J]=\frac{\partial\{\bar{x}\}}{\partial\{x\}}=\left[\frac{\bar{x}_{1}, \bar{x}_{2}, \bar{x}_{3}}{x_{1}, x_{2}, x_{3}}\right] ; \quad[\bar{J}]=\frac{\partial\{x\}}{\partial\{\bar{x}\}}=\left[\frac{x_{1}, x_{2}, x_{3}}{\bar{x}_{1}, \bar{x}_{2}, \bar{x}_{3}}\right]
$$

in which the columns of $[J]$ are covariant base vectors $\tilde{\boldsymbol{g}}_{i}$, whereas the rows of $[\bar{J}]$ are contravariant base vectors $\tilde{\boldsymbol{g}}^{i}[38] .[J]$ and $[\bar{J}]$ are Jacobians of deformation tensors. The basis defined by $[\bar{J}]$ is reciprocal to the basis defined by $[J]$. The following relations are useful in the paper: 


$$
\begin{gathered}
\frac{D}{D t}[J]=[\bar{L}][J] \\
\frac{D}{D t}[\bar{J}]=-[\bar{J}][\bar{L}] \\
\text { where } \bar{L}_{i j}=\frac{\partial \bar{v}_{i}}{\partial \bar{x}_{j}}
\end{gathered}
$$

in which $\frac{D}{D t}$ stands for material derivative, $[\bar{L}]$ is the spatial velocity gradient tensor, and $\bar{v}_{i}$ are the velocity components of a material point $\bar{x}_{i}$ in the current configuration in the $x$-frame. Over bar on all dependent quantities refers to their Eulerian descriptions, i.e., they are functions of $\bar{x}_{i}$ and $t$ whereas the quantities without over bar are their Lagrangian description, i.e., they are functions of $x_{i}$ and $t$. Thus, $\bar{Q}(\overline{\boldsymbol{x}}, t)$ and $Q(\boldsymbol{x}, t)$ are Eulerian and Lagrangian description of a quantity $Q$ in the current configuration.

\section{Choices of Stress Tensor, Moment Tensor, and Convected Time Derivatives of the Strain Tensor}

Stress, moment, and strain tensors and their convected time derivatives can be considered in contravariant basis, covariant basis, or Jaumann rates. Following reference [38] for example $\left(\overline{\boldsymbol{\sigma}}^{(0)}, \overline{\boldsymbol{\sigma}}_{(0)},{ }^{(0)} \overline{\boldsymbol{\sigma}}^{J}\right),\left(\overline{\boldsymbol{m}}^{(0)}, \overline{\boldsymbol{m}}_{(0)},{ }^{(0)} \overline{\boldsymbol{m}}^{J}\right)$ can be considered as measures of Cauchy stress and moment tensors in contravariant and covariant basis and corresponding to Jaumann rates. Likewise we can consider $\left[\gamma^{(k)}\right],\left[\gamma_{(k)}\right],\left[{ }^{(k)} \gamma^{J}\right] ; k=1,2, \cdots, n_{r}$, the convected time derivatives of the Almansi, Green's strain tensor and Jaumann rates. Where, $\left[\gamma^{(1)}\right]=\left[\gamma_{(1)}\right]=\left[{ }^{(1)} \gamma^{J}\right]=[\bar{D}]$, symmetric part of the velocity gradient tensor. Let ${ }^{(0)} \overline{\boldsymbol{\sigma}},{ }^{(0)} \overline{\boldsymbol{m}}$, and $\left[{ }^{(k)} \gamma\right] ; k=1,2, \cdots, n_{r}$ define Cauchy stress tensor, Cauchy moment tensor and convected time derivatives of the strain tensor conjugate to the stress tensor in a chosen basis. Conjugate measure to ${ }^{(0)} \overline{\boldsymbol{m}}$ is yet to be decided. We present derivations of the constitutive theories using this notation so that the resulting derivations are basis independent. By replacing $\left({ }^{(0)} \overline{\boldsymbol{\sigma}},{ }^{(0)} \overline{\boldsymbol{m}},\left[{ }^{(k)} \gamma\right] ; k=1,2, \cdots, n_{r}\right) \quad$ with $\quad\left(\overline{\boldsymbol{\sigma}}^{(0)}, \overline{\boldsymbol{m}}^{(0)},\left[\gamma_{(k)}\right] ; k=1,2, \cdots, n_{r}\right)$, $\left(\overline{\boldsymbol{\sigma}}_{(0)}, \overline{\boldsymbol{m}}_{(0)},\left[\gamma^{(k)}\right] ; k=1,2, \cdots, n_{r}\right)$, and $\left({ }^{(0)} \overline{\boldsymbol{\sigma}}^{J},{ }^{(0)} \overline{\boldsymbol{m}}^{J},\left[{ }^{(k)} \gamma^{J}\right] ; k=1,2, \cdots, n_{r}\right)$, the constitutive theories in contravariant basis, covariant basis, and in Jaumann rates can be obtained. Since in this paper we consider non-classical polymeric fluids, we also need to consider convected time derivatives of the Cauchy stress tensor as well as Cauchy moment tensor. Let ${ }^{(i)} \overline{\boldsymbol{\sigma}} ; i=1,2, \cdots, n_{\sigma}$ and ${ }^{(j)} \overline{\boldsymbol{m}} ; j=1,2, \cdots, n_{m}$ be the convected time derivatives of Cauchy stress and moment tensors up to orders $n_{\sigma}$ and $n_{m}$. Additionally, rotation rates and their gradients naturally appear in the balance laws through thermodynamic equilibrium considerations. As shown subsequently, the heat vector can be chosen to be basis independent if its argument tensors are density, temperature gradient, and temperature. 


\section{Rotation Rates, Gradients of Rotation Rates and Decompositions}

Let $[\bar{L}]$ be the velocity gradient tensor, then

$$
[\bar{L}]=\left[\frac{\partial\{\bar{v}\}}{\partial\{\bar{x}\}}\right]=[\bar{D}]+[\bar{W}]
$$

$[\bar{D}]$ and $[\bar{W}]$ are symmetric and antisymmetric tensors

$$
[\bar{D}]=\frac{1}{2}\left([\bar{L}]+[\bar{L}]^{\mathrm{T}}\right) ; \quad[\bar{W}]=\frac{1}{2}\left([\bar{L}]-[\bar{L}]^{\mathrm{T}}\right)
$$

$[\bar{W}]$ is internal rotation rate tensor containing rotation angle rates about $x_{1}, x_{2}, x_{3}$ axes in the $x$-frame. If we define rotation angle rates ${ }_{i}^{t} \bar{\Theta}_{x 1},{ }_{i}^{t} \bar{\Theta}_{x 2},{ }_{i}^{t} \bar{\Theta}_{x 3}$ by

$$
\begin{aligned}
& { }_{i}^{t} \bar{\Theta}_{x 1}={ }_{i}^{t} \bar{\Theta}_{1}=\frac{1}{2}\left(\frac{\partial \bar{v}_{2}}{\partial \bar{x}_{3}}-\frac{\partial \bar{v}_{3}}{\partial \bar{x}_{2}}\right) \\
& { }_{i}^{t} \bar{\Theta}_{x 2}={ }_{i}^{t} \bar{\Theta}_{2}=\frac{1}{2}\left(\frac{\partial \bar{v}_{3}}{\partial \bar{x}_{1}}-\frac{\partial \bar{v}_{1}}{\partial \bar{x}_{3}}\right) \\
& { }_{i}^{t} \bar{\Theta}_{x 3}={ }_{i}^{t} \bar{\Theta}_{3}=\frac{1}{2}\left(\frac{\partial \bar{v}_{1}}{\partial \bar{x}_{2}}-\frac{\partial \bar{v}_{2}}{\partial \bar{x}_{1}}\right)
\end{aligned}
$$

then,

$$
[\bar{W}]=\left[\begin{array}{ccc}
0 & { }_{i}^{t} \bar{\Theta}_{x 3} & -{ }_{i}^{t} \bar{\Theta}_{x 2} \\
-{ }_{i}^{t} \bar{\Theta}_{x 3} & 0 & { }_{i}^{t} \bar{\Theta}_{x 1} \\
{ }_{i}^{t} \bar{\Theta}_{x 2} & -{ }_{i}^{t} \bar{\Theta}_{x 1} & 0
\end{array}\right]
$$

If we represent rotation angle rates as a vector $\left\{{ }_{i}^{t} \bar{\Theta}\right\}$

$$
\left\{{ }_{i}^{t} \bar{\Theta}\right\}^{\mathrm{T}}=\left[{ }_{i}^{t} \bar{\Theta}_{x 1},{ }_{i}^{t} \bar{\Theta}_{x 2},{ }_{i}^{t} \bar{\Theta}_{x 3}\right]
$$

Alternatively, we could consider

$$
\bar{\nabla} \times \overline{\boldsymbol{v}}=\boldsymbol{e}_{1}\left(-2\left({ }_{i}^{t} \bar{\Theta}_{x 1}\right)\right)+\boldsymbol{e}_{2}\left(-2\left({ }_{i}^{t} \bar{\Theta}_{x 2}\right)\right)+\boldsymbol{e}_{3}\left(-2\left({ }_{i}^{t} \bar{\Theta}_{x 3}\right)\right)
$$

rotation angle rates in (4.3) are positive when clockwise and correspond to half the angles whereas the rotation angle rates (coefficients of $\boldsymbol{e}_{i}$ ) are positive when considered counterclockwise and correspond to full angles. We note that ${ }_{i}^{t} \bar{\Theta}$ are purely due to the velocity gradient tensor $\overline{\boldsymbol{L}}$ hence, are present in all deforming fluent continua. We refer to these ${ }_{i}^{t} \bar{\Theta}$ as internal rotation angle rates. The gradients of $\left\{{ }_{i}^{t} \bar{\Theta}\right\}$ in (4.5) can be defined as

$$
\left[{ }_{i}^{t} \Theta \bar{J}\right]=\left[\frac{\partial\left\{{ }_{i}^{t} \bar{\Theta}\right\}}{\partial\{\bar{x}\}}\right]=\left[{ }_{i}^{t} \Theta \bar{J}\right]+\left[{ }_{i}^{t} \Theta \bar{J}\right]
$$

In which $\left[{ }_{s}^{t} \bar{J}\right]$ and $\left[{ }_{i}^{t \Theta} \bar{J}\right]$ are symmetric and antisymmetric components of the gradients of rotation rate tensor $\left[{ }_{i}^{t} \Theta \bar{J}\right]$ and are defined as

$$
\begin{aligned}
& {\left[{ }_{i}^{t} \Theta \bar{J}\right]=\frac{1}{2}\left(\left[{ }_{i}^{t} \Theta \bar{J}\right]+\left[{ }_{i}^{t} \Theta \bar{J}\right]^{\mathrm{T}}\right)} \\
& {\left[{ }_{i}^{t} \Theta \bar{J}\right]=\frac{1}{2}\left(\left[{ }_{i}^{t} \Theta \bar{J}\right]-\left[{ }_{i}^{t} \Theta \bar{J}\right]^{\mathrm{T}}\right)}
\end{aligned}
$$




\section{Conservation and Balance Laws}

Consider a tetrahedron of volume $V$ and boundary $\partial V$ in the reference configuration whose planes are parallel to the fixed $x$-frame and whose oblique plane is subjected to average stress $\overline{\boldsymbol{P}}$ and average moment $\overline{\boldsymbol{M}}$. Upon deformation it occupies volume $\bar{V}$ with boundary surface $\partial \bar{V}$. The faces (planes) of the deformed tetrahedron are defined by covariant base vectors. These tetrahedron faces are flat but not orthogonal to each other as well as not parallel to the planes of the $x$-frame. Equilibrium considerations for the deformed tetrahedron yield the conservation and the balance laws keeping in mind that these laws resulting from classical continuum theories may need to be modified to the existence of the new physics associated with internal rotation rates and conjugate Cauchy moment tensor ${ }^{(0)} \overline{\boldsymbol{m}}$ that balances with $\overline{\boldsymbol{M}}$ through Cauchy principle. Conservation of mass, balance of linear momenta, balance of angular momenta, first and second laws of thermodynamics yield the following [1] [2] [3] [4] in the current configuration.

$$
\begin{aligned}
& \frac{\partial \bar{\rho}}{\partial t}+\bar{\nabla} \cdot(\overline{\rho v})=0 \\
& \bar{\rho} \frac{\partial \bar{v}_{i}}{\partial t}+\bar{\rho} \bar{v}_{j} \frac{\partial \bar{v}_{i}}{\partial \bar{x}_{j}}-\bar{\rho} \bar{F}_{i}^{b}-\frac{\partial\left({ }^{(0)} \bar{\sigma}_{j i}\right)}{\partial \bar{x}_{j}}=0 \\
& { }^{(0)} \bar{m}_{p k, p}-\epsilon_{i j k}{ }^{(0)} \bar{\sigma}_{i j}=0 \\
& \bar{\rho} \frac{D \bar{e}}{D t}+\bar{\nabla} \cdot \overline{\boldsymbol{q}}-\operatorname{tr}\left(\left[{ }^{(0)} \bar{\sigma}\right][\bar{L}]\right)-\operatorname{tr}\left(\left[{ }^{(0)} \bar{m}\right]\left[{ }^{t} \Theta \bar{J}\right]\right)-{ }_{i}^{t} \bar{\Theta} \cdot\left(\epsilon:^{(0)} \overline{\boldsymbol{\sigma}}\right)=0 \\
& \bar{\rho}\left(\frac{D \bar{\Phi}}{D t}+\bar{\eta} \frac{D \bar{\theta}}{D t}\right)+\frac{\overline{\boldsymbol{q}} \cdot \overline{\boldsymbol{g}}}{\bar{\theta}}-\operatorname{tr}\left(\left[{ }^{(0)} \overline{\boldsymbol{\sigma}}\right][\bar{L}]\right)-\operatorname{tr}\left(\left[{ }^{(0)} \bar{m}\right]\left[{ }_{i}^{t} \Theta \bar{J}\right]\right)-{ }_{i}^{t} \bar{\Theta} \cdot\left(\epsilon:^{(0)} \overline{\boldsymbol{\sigma}}\right) \leq 0
\end{aligned}
$$

in which

$$
\operatorname{tr}\left(\left[{ }^{(0)} \bar{\sigma}\right][\bar{L}]\right)={ }^{(0)} \bar{\sigma}_{i j} \frac{\partial \bar{v}_{i}}{\partial \bar{x}_{j}} ; \quad \operatorname{tr}\left(\left[\left({ }^{(0)} \bar{m}\right]\left[{ }^{t} \Theta \bar{J}\right]\right)={ }^{(0)} \bar{m}_{k j} \frac{\partial\left({ }_{i}^{t} \bar{\Theta}_{k}\right)}{\partial \bar{x}_{j}}\right.
$$

The Cauchy stress tensor ${ }^{(0)} \overline{\boldsymbol{\sigma}}$ is nonsymmetric and so is the Cauchy moment tensor ${ }^{(0)} \overline{\boldsymbol{m}}$. Antisymmetric components of ${ }^{(0)} \overline{\boldsymbol{\sigma}}$ are balanced with the gradients of ${ }^{(0)} \overline{\boldsymbol{m}}$ (Equation (5.3)). $\bar{\rho}=\bar{\rho}(\overline{\boldsymbol{x}}, t)$ is density, $\bar{F}_{i}^{b}$ are body forces per unit mass, $\bar{e}$ is specific internal energy, $\overline{\boldsymbol{q}}$ is heat vector, $\bar{\Phi}$ is Helmholtz free energy density, $\bar{\eta}$ is entropy density, $\overline{\boldsymbol{g}}$ is temperature gradients tensor, and $\bar{\theta}$ is temperature.

\section{Balance of Moment of Moments Balance Law}

Yang et al. [75] showed that when the additional physics of internal rotations is accounted for in a deforming volume of solid matter the conservation and the balance laws used in classical continuum mechanics are not sufficient to ensure 
equilibrium of the deforming matter. Yang et al. [75] presented equilibrium of the moment of moments as an additional equilibrium law necessary in non-classical continuum mechanics. The derivation originates from geometric consideration and requires static balance of the moment of moments due to Cauchy moment tensor and the moment of moments due to antisymmetric part of the Cauchy stress tensor. This derivation in the strict sense cannot be called a balance law, i.e., we cannot refer to this as "balance of moment of moments balance law" as a balance law must be derived from rate considerations (as rate of linear and angular momenta in the balance laws of linear and angular momenta). Surana et al. [76] [77] have shown that in case of non-classical continuum mechanics considering internal rotations and rotation rates, rate of change of angular momenta due to rotation rates must balance with the moment of moments and the moment of moments due to antisymmetric Cauchy stress tensor. Since the rate of change of moment of angular momenta due to rotation rates is zero in continuum mechanics (as the material points have no inertia). Thus, the complete balance of moment of moments derivation in [76] [77] reduces to the same equation as derived by Yang et al. [75] using static equilibrium considerations. Based on this balance law

$$
\epsilon_{i j k}{ }^{(0)} \bar{m}_{i j}=0
$$

must hold. Equation (5.6) implies that ${ }^{(0)} \bar{m}_{i j}={ }^{(0)} \bar{m}_{j i}$, i.e., the Cauchy moment tensor is symmetric. On the other hand in the absence of this balance law, symmetry of the Cauchy moment tensor is not established, hence Cauchy moment tensor ${ }^{(0)} \overline{\boldsymbol{m}}$ will be nonsymmetric. In the derivation of the constitutive theory for ${ }^{(0)} \overline{\boldsymbol{m}}$ we assume ${ }^{(0)} \overline{\boldsymbol{m}}$ to be nonsymmetric implying that the balance of moment of moments is not considered as a balance law. This is the more general case. The constitutive theories when ${ }^{(0)} \overline{\boldsymbol{m}}$ is symmetric are a subset of the more general case in which ${ }^{(0)} \overline{\boldsymbol{m}}$ is not symmetric, keeping in mind that nonsymmetric part of ${ }^{(0)} \overline{\boldsymbol{m}}$, i.e., absence of this balance law leads to spurious behavior as shown in [76] [77].

\section{Conjugate Pairs in Entropy Inequality, Constitutive Variables and Their Argument Tensors}

From the entropy inequality we note that in each of the two trace terms both tensors are nonsymmetric, thus based on the works of Spencer, Wang and Zhang and others [61]-[73] these pairs of tensors in each trace term do not constitute conjugate pairs. That is either of the tensors in each pair cannot be expressed in terms of the other due to lack of existence of integrity or basis for nonsymmetric tensors. We consider the following

$$
\begin{aligned}
& \bar{\nabla} \cdot{ }^{(0)} \overline{\boldsymbol{m}}=\boldsymbol{\epsilon}:^{\left({ }^{0}\right)} \overline{\boldsymbol{\sigma}} \\
& {[\bar{L}]=[\bar{D}]+[\bar{W}]}
\end{aligned}
$$

Stress tensor, and moment tensor, and rotation rate gradient tensor decompo- 
sitions yield

$$
\left[{ }^{(0)} \bar{\sigma}\right]=\left[{ }_{s}^{(0)} \bar{\sigma}\right]+\left[{ }_{a}^{(0)} \bar{\sigma}\right] ;\left[{ }^{(0)} \bar{m}\right]=\left[{ }_{s}^{(0)} \bar{m}\right]+\left[{ }_{a}^{(0)} \bar{m}\right] \text { and }\left[{ }^{t} \Theta \bar{J}\right]=\left[{ }_{i}^{t} \Theta \bar{J}\right]+\left[{ }_{s}^{t}{ }_{a}^{t} \bar{J}\right]
$$

in which subscripts $s$ and a stand for symmetric and antisymmetric. Substituting from (6.1)-(6.3) in (5.5) and noting that

$$
\begin{aligned}
& \operatorname{tr}\left(\left[{ }_{s}^{(0)} \bar{\sigma}\right][\bar{W}]\right)=0 ; \quad \operatorname{tr}\left(\left[{ }_{a}^{(0)} \bar{\sigma}\right][\bar{D}]\right)=0 \\
& \operatorname{tr}\left(\left[\begin{array}{c}
(0) \\
s \\
s
\end{array}\right]\left[\begin{array}{c}
t \\
i \\
a
\end{array} \bar{J}\right]\right)=0 ; \quad \operatorname{tr}\left(\left[\begin{array}{c}
(0) \\
a \\
m
\end{array}\right]\left[\begin{array}{c}
{ }_{i}^{t} \Theta \\
s \\
s
\end{array}\right]\right)=0
\end{aligned}
$$

and

$$
\operatorname{tr}\left(\left[{ }_{a}^{(0)} \bar{\sigma}\right][\bar{W}]\right)=-{ }_{i}^{t} \overline{\boldsymbol{\Theta}} \cdot\left(\boldsymbol{\epsilon}:{ }^{(0)} \overline{\boldsymbol{\sigma}}\right)
$$

We obtain the following from (5.5)

$$
\begin{aligned}
& \bar{\rho}\left(\frac{D \bar{\Phi}}{D t}+\bar{\eta} \frac{D \bar{\theta}}{D t}\right)+\frac{\overline{\boldsymbol{q}} \cdot \overline{\boldsymbol{g}}}{\bar{\theta}}-\operatorname{tr}\left(\left[{ }_{s}^{(0)} \bar{\sigma}\right][\bar{D}]\right) \\
& -\operatorname{tr}\left(\left[\left[_{s}^{(0)} \bar{m}\right]\left[\left[{ }_{i}^{t}{ }_{s}^{t} \bar{J}\right]\right)-\operatorname{tr}\left(\left[{ }^{(0)} \bar{m}\right]\left[\left[_{i}^{t}{ }_{a} \bar{J}\right]\right) \leq 0\right.\right.\right.
\end{aligned}
$$

The energy equation can accordingly be written as

$$
\bar{\rho} \frac{D \bar{e}}{D t}+\bar{\nabla} \cdot \overline{\boldsymbol{q}}-\operatorname{tr}\left(\left[\begin{array}{c}
(0) \\
s
\end{array}\right][\bar{D}]\right)-\operatorname{tr}\left(\left[\begin{array}{c}
(0) \\
s
\end{array}\right]\left[\begin{array}{c}
{ }_{i}^{t} \Theta \\
s \\
s
\end{array}\right]\right)-\operatorname{tr}\left(\left[\begin{array}{c}
(0) \\
a
\end{array}\right]\left[\begin{array}{c}
{ }_{i} \Theta \\
i \\
a
\end{array}\right]\right)=0
$$

We note that in (6.6), in the trace terms either both tensors are symmetric or antisymmetric, hence all three trace terms in (6.6) can be considered as conjugate pairs in the constitutive theories. First, from (6.6) we can easily infer that $\bar{\Phi}, \bar{\eta}, \overline{\boldsymbol{q}},{ }_{s}^{(0)} \overline{\boldsymbol{\sigma}},{ }_{s}^{(0)} \overline{\boldsymbol{m}}$ and ${ }_{a}^{(0)} \overline{\boldsymbol{m}}$ are a possible choice of constitutive variables. The argument tensors of these constitutive variables are decided using the conjugate pairs as well as the desired physics these are to represent that perhaps may not be obvious from the entropy inequality.

For compressible matter, density varies during evolution. Based on continuity equation in Lagrangian description, this is defined by changing $|J|$

$$
\rho_{0}=|J| \rho(\boldsymbol{x}, t)
$$

Hence $|J|$ or $\frac{\rho_{0}}{\rho(\boldsymbol{x}, t)}$ or $\frac{1}{\rho(\boldsymbol{x}, t)}$, hence $\frac{1}{\bar{\rho}(\overline{\boldsymbol{x}}, t)}$ in Eulerian description must be an argument tensor of the constitutive variables. Choice of $\bar{\theta}$ as an argument tensor is straight forward. ${ }_{s}^{(0)} \overline{\boldsymbol{\sigma}}$ as a constitutive variable and $\overline{\boldsymbol{D}}$ as its argument tensor is obvious from the conjugate pair in the entropy inequality. Likewise ${ }_{s}^{(0)} \overline{\boldsymbol{m}}$ and ${ }_{a}^{(0)} \overline{\boldsymbol{m}}$ are constitutive variables and ${ }_{s}^{t \Theta} \boldsymbol{J}$ and ${ }_{a}^{t \Theta} \boldsymbol{J}$ are their argument tensors is straight forward as well. Similarly, $\overline{\boldsymbol{q}}$ as dependent variable and $\overline{\boldsymbol{g}}$ as its argument tensor is also quite obvious from (6.6). $\bar{\Phi}$ and $\bar{\eta}$ at this stage must contain totality of all argument tensors based on principle of equipresence some of which may be ruled out later due to some other considerations at a later stage in the derivation. Thus, at this stage we have 


$$
\begin{aligned}
& \bar{\Phi}=\bar{\Phi}\left(\frac{1}{\bar{\rho}}, \overline{\boldsymbol{D}},{ }_{s}^{t} \Theta \boldsymbol{J},{ }_{a}^{t} \Theta \boldsymbol{J}, \overline{\boldsymbol{g}}, \bar{\theta}\right) \\
& \bar{\eta}=\bar{\eta}\left(\frac{1}{\bar{\rho}}, \overline{\boldsymbol{D}},{ }_{s}^{t} \Theta \boldsymbol{J},{ }_{a}^{t} \Theta \boldsymbol{J}, \overline{\boldsymbol{g}}, \bar{\theta}\right) \\
& { }_{s}^{(0)} \overline{\boldsymbol{\sigma}}={ }_{s}^{(0)} \overline{\boldsymbol{\sigma}}\left(\frac{1}{\bar{\rho}}, \overline{\boldsymbol{D}}, \bar{\theta}\right) \\
& { }_{s}^{(0)} \overline{\boldsymbol{m}}={ }_{s}^{(0)} \overline{\boldsymbol{m}}\left(\frac{1}{\bar{\rho}},{ }_{s}^{t} \Theta \boldsymbol{J}, \bar{\theta}\right) \\
& { }_{a}^{(0)} \overline{\boldsymbol{m}}={ }_{a}^{(0)} \overline{\boldsymbol{m}}\left(\frac{1}{\bar{\rho}},{ }_{a}^{t} \Theta \boldsymbol{J}, \bar{\theta}\right) \\
& \overline{\boldsymbol{q}}=\overline{\boldsymbol{q}}\left(\frac{1}{\bar{\rho}}, \overline{\boldsymbol{g}}, \bar{\theta}\right)
\end{aligned}
$$

These argument tensors need to be modified based on the following remarks.

1) Recall that first convected time derivative of the Green's strain tensor in covariant basis is $[\bar{D}]$, i.e.,

$$
[\bar{D}]=\left[\gamma_{(1)}\right]
$$

Tensor $\gamma_{(1)}$ is a fundamental kinematic tensor in covariant basis based on Green's strain tensor, a covariant measure.

2) Likewise if we consider convected time derivative of Almansi strain tensor in contravariant basis, then $[\bar{D}]$ is also the convected time derivative of the Almansi strain tensor in contravariant basis, i.e.,

$$
[\bar{D}]=\left[\gamma^{(1)}\right]
$$

Tensor $\left[\gamma^{(1)}\right]$ is also a fundamental kinematic tensor in contravariant basis.

3) We also know that [38]

$$
\left[{ }^{(1)} \gamma^{J}\right]=\left[\gamma^{(1)}\right]=\left[\gamma_{(1)}\right]=[\bar{D}]
$$

in which $\left[{ }^{(1)} \gamma^{J}\right]$ is Jaumann rate.

4) Convected time derivatives of Green's and Almansi strain tensors of orders higher than one (hence Jaumann rates as well) can be derived [38]. These are all fundamental kinematic tensors as well.

$$
\begin{aligned}
& {\left[\gamma^{(j)}\right] ; j=1,2, \cdots, n} \\
& {\left[\gamma_{(j)}\right] ; j=1,2, \cdots, n} \\
& {\left[{ }^{(j)} \gamma^{J}\right] ; j=1,2, \cdots, n}
\end{aligned}
$$

Thus, $[\bar{D}]$ in (6.9) can be replaced by $\left[{ }^{(j)} \gamma\right] ; j=1,2, \cdots, n$ in which ${ }^{(j)} \gamma$ (for basis independence considerations of the derivation) can be a desired choice from (6.13).

5) By examining the Maxwell, Oldroyd-B, and Giesekus constitutive models for polymeric fluids (based on classical mechanics), we note that these contain convected time derivatives of orders one and zero (same as stress tensor) of the 
stress tensor. In the work presented here we generalize this choice, hence consider convected time derivatives of Cauchy stress tensor up to orders $m$ in co- and contra-variant bases as well as Jaumann rates, i.e., we choose ${ }_{s}^{(j)} \overline{\boldsymbol{\sigma}} ; j=0,1, \cdots, m$ (due to basis independence of the derivation). Thus, now we can update the choice of constitutive variables and their argument tensors in (6.9). We replace $[\bar{D}]$ with $\left[{ }^{(j)} \gamma\right] ; j=1,2, \cdots, n$ and ${ }_{s}^{(0)} \overline{\boldsymbol{\sigma}}$ by ${ }_{s}^{(m)} \overline{\boldsymbol{\sigma}}$. Additionally, ${ }_{s}^{\left({ }^{(j)}\right.} \overline{\boldsymbol{\sigma}} ; j=0,1, \cdots, m-1$ become argument tensors of ${ }_{s}^{(m)} \bar{\sigma}$.

6) Parallel to the Cauchy stress tensor, we must also consider $\left[{ }_{s}^{(i)} \bar{m}\right] ; i=1,2, \cdots,{ }^{1} m,\left[{ }_{a}^{(j)} \bar{m}\right] ; j=1,2, \cdots,{ }^{2} m$ and replace ${ }_{s}^{(0)} \overline{\boldsymbol{m}}$ and ${ }_{a}^{(0)} \overline{\boldsymbol{m}}$ in (6.9) by $\left[\begin{array}{c}\left({ }^{1} m\right) \\ s\end{array} \bar{m}\right]$ and $\left[\begin{array}{c}\left({ }^{2} m\right) \\ a\end{array} \bar{m}\right]$. Additionally, we must also include $\left[{ }_{s}^{(i)} \bar{m}\right] ; i=0,1, \cdots,\left({ }^{1} m-1\right)$ and $\left[{ }_{a}^{(j)} \bar{m}\right] ; j=0,1, \cdots,\left({ }^{2} m-1\right)$ as argument tensors of $\left[{ }_{s}^{\left({ }^{1} m\right)} \bar{m}\right]$ and $\left[\underset{a}{\left({ }^{2} m\right)} \bar{m}\right]$.

Based on remarks (1)-(6) we can modify the choice of constitutive variables and their argument tensors in (6.9). Keeping in mind that at this stage $\bar{\Phi}$ and $\bar{\eta}$ must include totality of all argument tensors.

$$
\begin{aligned}
& \bar{\Phi}=\bar{\Phi}\left(\frac{1}{\bar{\rho}},{ }^{(i)} \gamma ; i=1,2, \cdots, n,{ }_{s}^{(j)} \overline{\boldsymbol{\sigma}} ; j=0,1, \cdots,(m-1),{ }_{s}^{(k)} \overline{\boldsymbol{m}} ;\right. \\
& \left.k=0,1, \cdots,\left({ }^{1} m-1\right),{ }_{a}^{(l)} \overline{\boldsymbol{m}} ; l=0,1, \cdots,\left({ }^{2} m-1\right),{ }_{s}^{t \Theta} \boldsymbol{J},{ }_{a}^{t} \Theta \quad J, \overline{\boldsymbol{g}}, \bar{\theta}\right) \\
& \bar{\eta}=\bar{\eta}\left(\frac{1}{\bar{\rho}},{ }^{(i)} \boldsymbol{\gamma} ; i=1,2, \cdots, n,{ }_{s}^{(j)} \overline{\boldsymbol{\sigma}} ; j=0,1, \cdots,(m-1),{ }_{s}^{(k)} \overline{\boldsymbol{m}} ;\right.
\end{aligned}
$$

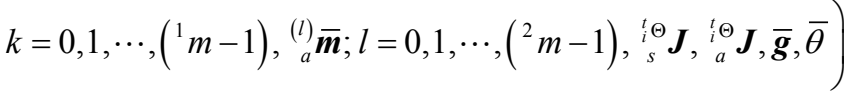

$$
\begin{aligned}
& { }_{s}^{(m)} \overline{\boldsymbol{\sigma}}={ }_{s}^{(m)} \overline{\boldsymbol{\sigma}}\left(\frac{1}{\bar{\rho}},{ }^{(i)} \gamma ; i=1,2, \cdots, n,{ }_{s}^{(j)} \overline{\boldsymbol{\sigma}} ; j=0,1, \cdots,(m-1), \bar{\theta}\right) \\
& { }_{s}^{\left({ }^{1}\right)} \overline{\boldsymbol{m}}={ }_{s}^{\left({ }^{1}\right)} \overline{\boldsymbol{m}}\left(\frac{1}{\bar{\rho}},{ }_{s}^{t \Theta} \boldsymbol{J},{ }_{s}^{(k)} \overline{\boldsymbol{m}} ; k=0,1, \cdots,\left({ }^{1} m-1\right), \bar{\theta}\right) \\
& { }_{a}^{\left({ }^{2}\right)} \overline{\boldsymbol{m}}={ }_{a}^{\left({ }^{2}\right)} \overline{\boldsymbol{m}}\left(\frac{1}{\bar{\rho}},{ }_{a}^{t}{ }_{a} \boldsymbol{J},{ }_{a}^{(l)} \overline{\boldsymbol{m}} ; l=0,1, \cdots,\left({ }^{2} m-1\right), \bar{\theta}\right) \\
& \overline{\boldsymbol{q}}=\overline{\boldsymbol{q}}\left(\frac{1}{\bar{\rho}}, \overline{\mathrm{g}}, \bar{\theta}\right)
\end{aligned}
$$

using $\bar{\Phi}(\cdot)$ in (6.14) we can determine $\dot{\bar{\Phi}}$

$$
\begin{aligned}
\frac{D}{D t} \bar{\Phi}= & \dot{\bar{\Phi}}=\frac{\partial \bar{\Phi}}{\partial\left(\frac{1}{\bar{\rho}}\right)}\left(-\frac{1}{\bar{\rho}^{2}}\right) \dot{\bar{\rho}}+\sum_{j=1}^{n} \frac{\partial \bar{\Phi}}{\partial^{(j)} \gamma_{i k}}{ }^{(j)} \dot{\gamma}_{i k}+\sum_{j=0}^{(m-1)} \frac{\partial \bar{\Phi}}{\partial_{s}^{(j)} \bar{\sigma}_{i k}}{ }_{s}^{(j)} \dot{\bar{\sigma}}_{i k} \\
& +\sum_{j=0}^{\left({ }^{1}{ }^{m-1}\right)} \frac{\partial \bar{\Phi}}{\partial^{(j)} \bar{m}_{i k}}{ }_{s}^{(j)} \dot{\bar{m}}_{i k}+\sum_{j=0}^{\left({ }^{2}{ }^{m-1}\right)} \frac{\partial \bar{\Phi}}{\partial_{a}^{(j)} \bar{m}_{i k}}{ }_{a}^{(j)} \dot{\bar{m}}_{i k}+\frac{\partial \bar{\Phi}}{\partial \bar{g}_{i}} \dot{\bar{g}}_{i}+\frac{\partial \bar{\Phi}}{\partial \bar{\theta}} \dot{\bar{\theta}}
\end{aligned}
$$

From continuity equation 


$$
\begin{gathered}
\dot{\bar{\rho}}=-\bar{\rho} \overline{\boldsymbol{\nabla}} \cdot \overline{\boldsymbol{v}}=-\bar{\rho} \bar{D}_{k k}=-\bar{\rho} \bar{D}_{i k} \delta_{i k} \\
\text { and } \frac{\partial \bar{\Phi}}{\partial\left(\frac{1}{\bar{\rho}}\right)}=-\bar{\rho}^{2} \frac{\partial \bar{\Phi}}{\partial \bar{\rho}}
\end{gathered}
$$

Using (6.16) and (6.17) in (6.15) we can write

$$
\begin{aligned}
\dot{\bar{\Phi}}= & -\bar{\rho} \frac{\partial \bar{\Phi}}{\partial(\bar{\rho})} \bar{D}_{i k} \delta_{i k}+\sum_{j=1}^{n} \frac{\partial \bar{\Phi}}{\partial^{(j)} \gamma_{i k}}{ }^{(j)} \dot{\gamma}_{i k}+\sum_{j=0}^{(m-1)} \frac{\partial \bar{\Phi}}{\partial_{s}^{(j)} \bar{\sigma}_{i k}}{ }_{s}^{(j)} \dot{\bar{\sigma}}_{i k} \\
& +\sum_{j=0}^{\left({ }^{1} m-1\right)} \frac{\partial \bar{\Phi}}{\partial_{s}^{(j)} \bar{m}_{i k}}{ }_{s}^{(j)} \dot{\bar{m}}_{i k}+\sum_{j=0}^{\left({ }^{2}{ }^{m-1}\right)} \frac{\partial \bar{\Phi}}{\partial^{(j)} \bar{m}_{i k}}{ }_{a}^{(j)} \dot{\bar{m}}_{i k}+\frac{\partial \bar{\Phi}}{\partial \bar{g}_{i}} \dot{\bar{g}}_{i}+\frac{\partial \bar{\Phi}}{\partial \bar{\theta}}
\end{aligned}
$$

Substituting $\dot{\bar{\Phi}}$ from (6.18) in the entropy inequality (6.6) (using Einstein's notation for the trace terms) and regrouping the terms.

$$
\begin{aligned}
& \left(-\bar{\rho}^{2} \frac{\partial \bar{\Phi}}{\partial \bar{\rho}} \delta_{i k}-{ }_{s}^{(0)} \bar{\sigma}_{i k}\right) \bar{D}_{i k}+\bar{\rho} \sum_{j=1}^{n} \frac{\partial \bar{\Phi}}{\partial^{(j)} \gamma_{i k}}{ }^{(j)} \dot{\gamma}_{i k}+\bar{\rho} \sum_{j=0}^{(m-1)} \frac{\partial \bar{\Phi}}{\partial^{(j)} \bar{\sigma}_{i k}}{ }_{s}^{(j)} \dot{\bar{\sigma}}_{i k} \\
& +\bar{\rho} \sum_{j=0}^{\left({ }^{1} m-1\right)} \frac{\partial \bar{\Phi}}{\partial^{(j)} \bar{m}_{i k}}{ }_{s}^{(j)} \dot{\bar{m}}_{i k}+\bar{\rho} \sum_{j=0}^{\left({ }^{2}{ }^{m-1}\right)} \frac{\partial \bar{\Phi}}{\partial^{(j)} \bar{m}_{i k}}{ }_{a}^{(j)} \dot{\bar{m}}_{i k}+\bar{\rho} \frac{\partial \bar{\Phi}}{\partial \bar{g}_{i}} \dot{\bar{g}}_{i} \\
& +\bar{\rho}\left(\frac{\partial \bar{\Phi}}{\partial \bar{\theta}}+\bar{\eta}\right) \dot{\bar{\theta}}+\frac{\overline{\boldsymbol{q}} \cdot \overline{\boldsymbol{g}}}{\bar{\theta}}-{ }_{s}^{(0)} \bar{m}_{j k}\left({ }_{i}^{t} \bar{J}_{j k}\right)-{ }_{a}^{(0)} \bar{m}_{j k}\left({ }_{i}^{t}{ }_{a}^{t} \bar{J}_{j k}\right) \leq 0
\end{aligned}
$$

For (6.19) to hold for arbitrary but admissible ${ }^{(j)} \dot{\gamma} ; j=1,2, \cdots, n$, ${ }_{\stackrel{s}{(j)}} \dot{\overline{\boldsymbol{\sigma}}} ; j=0,1, \cdots,(m-1), \quad{ }_{s}^{(j)} \dot{\overline{\boldsymbol{m}}} ; j=0,1, \cdots,\left({ }^{1} m-1\right), \quad{ }_{a}^{(j)} \dot{\overline{\boldsymbol{m}}} ; j=0,1, \cdots,\left({ }^{2} m-1\right)$, $\dot{\bar{\theta}}$, and $\dot{\overline{\boldsymbol{g}}}$, the following must hold.

$$
\begin{gathered}
\bar{\rho} \frac{\partial \bar{\Phi}}{\partial^{(j)} \gamma_{i k}}=0 \Rightarrow \frac{\partial \bar{\Phi}}{\partial^{(j)} \gamma_{i k}}=0 ; j=1,2, \cdots, n \\
\bar{\rho} \frac{\partial \bar{\Phi}}{\partial_{s}^{(j)} \bar{\sigma}_{i k}}=0 \Rightarrow \frac{\partial \bar{\Phi}}{\partial^{(j)} \bar{\sigma}_{i k}}=0 ; j=0,1, \cdots,(m-1) \\
\bar{\rho} \frac{\partial \Phi}{\partial_{s}^{(j)} \bar{m}_{i k}}=0 \Rightarrow \frac{\partial \Phi}{\partial^{(j)} \bar{m}_{i k}}=0 ; j=0,1, \cdots,\left({ }^{1} m-1\right) \\
\bar{\rho} \frac{\partial \bar{\Phi}}{\partial_{a}^{(j)} \bar{m}_{i k}}=0 \Rightarrow \frac{\partial \bar{\Phi}}{\partial^{(j)} \bar{m}_{i k}}=0 ; j=0,1, \cdots,\left({ }^{2} m-1\right) \\
\bar{\rho}\left(\frac{\partial \bar{\Phi}}{\partial \bar{\theta}}+\bar{\eta}\right)=0 \Rightarrow \frac{\partial \bar{\Phi}}{\partial \bar{\theta}}+\bar{\eta}=0 \\
\bar{\rho} \frac{\partial \bar{\Phi}}{\partial \bar{g}_{i}}=0 \Rightarrow \frac{\partial \bar{\Phi}}{\partial \bar{g}_{i}}=0
\end{gathered}
$$

and the resulting entropy inequality (6.6) can be written as

$$
\left(-\bar{\rho}^{2} \frac{\partial \bar{\Phi}}{\partial \bar{\rho}} \delta_{i k}-{ }_{s}^{(0)} \bar{\sigma}_{i k}\right) \bar{D}_{i k}+\frac{\overline{\boldsymbol{q}} \cdot \overline{\boldsymbol{g}}}{\bar{\theta}}-{ }_{s}^{(0)} \bar{m}_{j k}\left({ }_{i}^{t} \Theta \bar{J}_{j k}\right)-{ }_{a}^{(0)} \bar{m}_{j k}\left({ }_{i}^{t} \Theta \bar{J}_{j k}\right) \leq 0
$$

Conditions (6.20)-(6.25) and entropy inequality (6.26) are fundamental relations.

\section{Remarks}


1) From (6.20) we note that $\bar{\Phi}$ is not a function of ${ }^{(j)} \gamma ; j=1,2, \cdots, n$.

2) From (6.21) we can conclude that $\bar{\Phi}$ is not a function of

${ }_{s}^{(j)} \overline{\boldsymbol{\sigma}} ; j=0,1, \cdots,(m-1)$.

3) Equations (6.22) imply that $\bar{\Phi}$ is not a function of

${ }_{s}^{(j)} \overline{\boldsymbol{m}} ; j=0,1, \cdots,\left({ }^{1} m-1\right)$.

4) From (6.23) we note that $\bar{\Phi}$ is not a function of

${ }_{a}^{(j)} \overline{\boldsymbol{m}} ; j=0,1, \cdots,\left({ }^{2} m-1\right)$.

5) From (6.24), $\bar{\eta}=-\frac{\partial \bar{\Phi}}{\partial \bar{\theta}}$, hence $\bar{\eta}$ is deterministic from $\bar{\Phi}$. Thus, $\bar{\eta}$ cannot be a constitutive variable.

6) Equations (6.25) imply that $\bar{\Phi}$ is not a function of $\overline{\boldsymbol{g}}$.

7) Lastly, in the entropy inequality (6.26), the following are admissible

$$
\begin{gathered}
\frac{\overline{\boldsymbol{q}} \cdot \overline{\boldsymbol{g}}}{\bar{\theta}} \leq 0 \\
{ }_{s}^{(0)} \bar{m}_{j k}\left({ }_{s}^{t} \Theta \bar{J}_{j k}\right) \geq 0 \\
{ }_{a}^{(0)} \bar{m}_{j k}\left({ }_{i}^{t}{ }_{a}^{t} \bar{J}_{j k}\right) \geq 0
\end{gathered}
$$

Condition (6.27) must be satisfied by the constitutive theory for $\overline{\boldsymbol{q}}$. Conditions (6.28) and (6.29) imply that the rate of work due to symmetric and antisymmetric parts of the Cauchy moment tensor must be positive.

8) The argument tensors of $\bar{\Phi}$ (based on (6.20)-(6.25)) are given by (using $\bar{\rho}$ in place of $\frac{1}{\bar{\rho}}$ )

$$
\bar{\Phi}=\bar{\Phi}(\bar{\rho}, \bar{\theta})
$$

Based on (6.30), the coefficient of $\bar{D}_{i k}$ in the entropy inequality (6.26) cannot be set to zero because this would imply that ${ }_{s}^{(0)} \bar{\sigma}$ is deterministic from $\bar{\Phi}$ which is only a function of $\bar{\rho}$ and $\bar{\theta}$. This is obviously not true based on the argument tensors of ${ }_{s}^{(0)} \bar{\sigma}$ in (6.14). Thus, at this stage the entropy inequality (49) must remain in this form.

\section{Constitutive Theories}

We consider entropy inequality (6.26) and introduce decomposition of ${ }_{s}^{(0)} \bar{\sigma}$ into

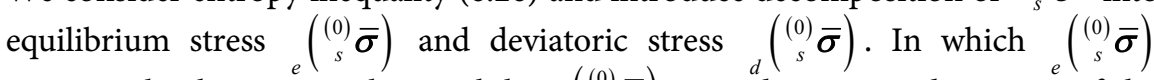
causes only change in volume while ${ }_{d}\left({ }_{s}^{(0)} \bar{\sigma}\right)$ introduces pure distortion of the volume of matter [38].

$$
{ }_{s}^{(0)} \bar{\sigma}={ }_{e}\left({ }_{s}^{(0)} \bar{\sigma}\right)+{ }_{d}\left({ }_{s}^{(0)} \bar{\sigma}\right)
$$

$\left({ }_{s}^{(0)} \bar{\sigma}\right)$ can only be a function of $\bar{\rho}$ and $\bar{\theta}$ [38]. Thus, we have the following, noting that ${ }_{d}\left({ }_{s}^{\left({ }^{\circ}\right)} \bar{\sigma}\right)$ cannot be a function of ${ }_{e}\left({ }_{s}^{(0)} \bar{\sigma}\right)$.

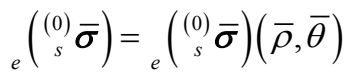

$$
\begin{aligned}
& \left.{ }_{d}\left({ }_{s}^{(m)} \bar{\sigma}\right)={ }_{d}\left({ }_{s}^{(m)} \bar{\sigma}\right)\left(\bar{\rho},{ }^{(j)} \gamma ; j=1,2, \cdots, n,{ }_{d}\left({ }_{s}^{(i)} \overline{\boldsymbol{\sigma}}\right) ; i=0,1, \cdots,(m-1), \bar{\theta}\right)\right\} \\
& \bar{\Phi}=\bar{\Phi}(\bar{\rho}, \bar{\theta})
\end{aligned}
$$


all the other constitutive variables and their argument tensors remain the same as defined by (6.14), except that $\frac{1}{\bar{\rho}}$ has been replaced by $\bar{\rho}$ [38]. Substituting (7.1) in the entropy inequality (6.26) and grouping terms.

$$
\begin{aligned}
& \left.\left(-\bar{\rho}^{2} \frac{\partial \bar{\Phi}}{\partial \bar{\rho}} \delta_{i k}-{ }_{e}\left({ }_{s}^{(0)} \bar{\sigma}_{i k}\right)\right) \bar{D}_{i k}-{ }_{d}{ }^{(0)} \bar{\sigma}_{i k}\right) \bar{D}_{i k}+\frac{\overline{\boldsymbol{q}} \cdot \overline{\boldsymbol{g}}}{\bar{\theta}} \\
& -{ }_{s}^{(0)} \bar{m}_{j k}\left({ }_{i}^{t}{ }_{s}^{t} \bar{J}_{j k}\right)-{ }_{a}^{(0)} \bar{m}_{j k}\left({ }_{i}^{t} \bar{J}_{j k}\right) \leq 0
\end{aligned}
$$

\subsection{Constitutive Theory for ${ }_{e}\left(\begin{array}{c}(0) \\ s\end{array} \bar{\sigma}\right)$ : Compressible Matter}

Based on (7.2), we can set the coefficient of $\bar{D}_{i k}$ in the first term in (7.3) to zero giving

$$
e^{\left({ }_{s}^{(0)} \bar{\sigma}_{i k}\right)}=-\bar{\rho}^{2} \frac{\partial \bar{\Phi}(\bar{\rho}, \bar{\theta})}{\partial \bar{\rho}} \delta_{i k}=\bar{p}(\bar{\rho}, \bar{\theta}) \delta_{i k}
$$

$\bar{p}(\bar{\rho}, \bar{\theta})$ is called thermodynamic pressure (defined by equation of state) and can be derived using $\bar{\Phi}$. If we assume compressive pressure to be positive, then $\bar{p}(\bar{\rho}, \bar{\theta})$ in (7.4) can be replaced by $-\bar{p}(\bar{\rho}, \bar{\theta})$. Equation (7.4) is the constitutive theory for the equilibrium part of the symmetric Cauchy stress tensor.

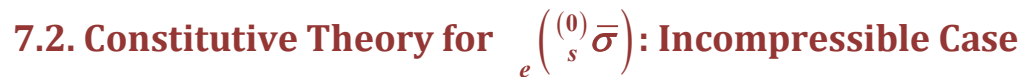

For incompressible fluent continua, $\bar{\rho}=\rho_{0}=$ constant , hence $\frac{\partial \bar{\Phi}}{\partial \bar{\rho}}=0$, thus for this case (7.4) cannot be used to derive constitutive theory for ${ }_{e}\left({ }_{s}^{(0)} \bar{\sigma}\right)$. For incompressible matter $|J|=1$, also

$$
\bar{\nabla} \cdot \overline{\boldsymbol{v}}=\operatorname{tr}[\bar{D}]=\bar{D}_{i k} \delta_{i k}=0
$$

This incompressibility condition must be enforced. Based on (7.5) we can write

$$
\bar{p}(\bar{\theta}) \bar{D}_{i k} \delta_{i k}=0
$$

In (7.6), $\bar{p}(\bar{\theta})$ is an arbitrary Lagrange multiplier. Adding (7.6) to entropy inequality (7.6) and setting $\frac{\partial \bar{\Phi}}{\partial \bar{\rho}}=0$, we obtain

$$
\left(\bar{p}(\bar{\theta}) \delta_{i k}-{ }_{e}\left({ }_{s}^{(0)} \bar{\sigma}_{i k}\right)\right) \bar{D}_{i k}-{ }_{d}\left({ }_{s}^{(0)} \bar{\sigma}_{i k}\right) \bar{D}_{i k}+\frac{\overline{\boldsymbol{q}} \cdot \overline{\boldsymbol{g}}}{\bar{\theta}}-{ }_{s}^{(0)} \bar{m}_{j k}\left({ }_{i}^{t} \Theta \bar{J}_{j k}\right)-{ }_{a}^{(0)} \bar{m}_{j k}\left({ }_{i j}^{t \Theta} \bar{J}_{j k}\right) \leq 0
$$

Setting the coefficient of $\bar{D}_{i k}$ in the first term to zero, we obtain the following constitutive theory for ${ }_{e}\left({ }_{s}^{(0)} \bar{\sigma}\right)$ for the incompressible case.

$$
\left.{ }_{e}^{\left({ }_{s}^{(0)}\right.} \bar{\sigma}_{i k}\right)=\bar{p}(\bar{\theta}) \delta_{i k}
$$

$\bar{p}(\bar{\theta})$ is called mechanical pressure. If we assume compressive pressure to be positive, then $\bar{p}(\bar{\theta})$ in (7.8) can be replaced by $-\bar{p}(\bar{\theta})$. The entropy inequa- 
lity now reduces to

$$
\frac{\overline{\boldsymbol{q}} \cdot \overline{\boldsymbol{g}}}{\bar{\theta}}-{ }_{d}\left({ }_{s}^{(0)} \bar{\sigma}_{i k}\right) \bar{D}_{i k}-{ }_{s}^{(0)} \bar{m}_{j k}\left({ }_{i}^{t \Theta} \bar{J}_{j k}\right)-{ }_{a}^{(0)} \bar{m}_{j k}\left({ }_{i}^{t \Theta} \bar{J}_{j k}\right) \leq 0
$$

The constitutive variables and their argument tensors now are as follows.

$$
\begin{aligned}
& \bar{\Phi}=\bar{\Phi}(\bar{\rho}, \bar{\theta}) \\
& { }_{d}\left({ }_{s}^{(m)} \overline{\boldsymbol{\sigma}}\right)={ }_{d}\left({ }_{s}^{(m)} \bar{\sigma}\right)\left(\bar{\rho},{ }^{(j)} \gamma ; j=1,2, \cdots, n,{ }_{d}\left(\begin{array}{c}
(k) \\
s
\end{array} \overline{\boldsymbol{\sigma}}\right) ; k=1,2, \cdots,(m-1), \bar{\theta}\right) \\
& { }_{s}^{\left({ }^{1}\right)} \overline{\boldsymbol{m}}={ }_{s}^{\left({ }^{1} m\right)} \overline{\boldsymbol{m}}\left(\bar{\rho},{ }_{i}^{t} \Theta \boldsymbol{J},{ }_{s}^{(k)} \overline{\boldsymbol{m}} ; k=0,1, \cdots,\left({ }^{1} m-1\right), \bar{\theta}\right) \\
& { }_{a}^{\left({ }^{2}\right)} \overline{\boldsymbol{m}}={ }_{a}^{\left({ }^{2}\right)} \overline{\boldsymbol{m}}\left(\bar{\rho},{ }_{a}^{t} \Theta \boldsymbol{J},{ }_{a}^{(k)} \overline{\boldsymbol{m}} ; k=0,1, \cdots,\left({ }^{2} m-1\right), \bar{\theta}\right) \\
& \overline{\boldsymbol{q}}=\overline{\boldsymbol{q}}(\bar{\rho}, \overline{\boldsymbol{g}}, \bar{\theta})
\end{aligned}
$$

\subsection{Constitutive Theory for ${ }_{d}\left({ }_{s}^{(0)} \bar{\sigma}\right)$}

We consider (7.11) defining the argument tensors of ${ }_{d}\left(\begin{array}{c}(m) \\ s\end{array} \bar{\sigma}\right)$. This constitutive theory has to be a rate theory in time in stress and strain rate tensors in order to incorporate dissipation mechanism as well as memory (rheology). We use representation theorem (or theory of generators and invariants) [29] [38] [61]-[73] to derive the constitutive theory for the deviatoric part of the symmetric Cauchy stress tensor. Let ${ }^{\sigma}{ }_{\sim}{ }^{i} ; i=1,2, \cdots, N_{s} \sigma$ be the combined generators of the argument tensors of $\left(\begin{array}{c}(m) \\ s\end{array} \bar{\sigma}\right)$ that are symmetric tensors of rank two and let ${ }^{\sigma}{ }_{\sim}{ }_{\sim}^{j} ; j=1,2, \cdots, M_{s} \sigma$ be the combined invariants of the same argument tensors, then using the representation theorem we can express ${ }_{d}\left({ }_{s}^{(m)} \bar{\sigma}\right)$ as a linear combination of ${ }^{\sigma}{ }_{\sim} \boldsymbol{G}^{i} ; i=1,2, \cdots, N_{s} \sigma$ and $\boldsymbol{I}$ in the current configuration.

$$
{ }_{d}\left({ }_{s}^{(m)} \overline{\boldsymbol{\sigma}}\right)={ }^{{ }^{\sigma} \sigma} \underset{\sim}{\alpha}(\boldsymbol{I})+\sum_{i=1}^{N_{s} \sigma}{ }_{s} \sigma{ }_{\sim}{ }^{i}\left({ }^{\sigma} \boldsymbol{G}_{\sim}^{i}\right)
$$

in which

$$
{ }^{\sigma} \alpha_{\sim}^{i}={ }^{\sigma}{ }_{\sim} \alpha^{i}\left(\bar{\rho},{ }^{s}{ }_{\sim} I^{j} ; j=1,2, \cdots, M_{s} \sigma, \bar{\theta}\right) ; i=0,1, \cdots, N_{s}
$$

To determine the material coefficients in (7.15), we expand each ${ }^{\sigma} \sigma \alpha_{\sim}^{i} ; i=0,1, \cdots, N_{s}$ in Taylor series in ${ }^{s}{ }_{\sim}^{I^{j}} ; j=1,2, \cdots, M_{s} \sigma$ and $\bar{\theta}$ about a known configuration $\underline{\Omega}$, retaining only up to linear terms in ${ }^{\sigma}{ }_{\sim}{ }_{\sim}^{j} ; j=1,2, \cdots, M_{s} \sigma$ and $\bar{\theta}$ (for simplicity) and then we substitute these ${ }_{s}^{\sigma}{\underset{\sim}{\alpha}}_{\sim}^{i} ; i=0,1, \cdots, N_{s \sigma}$ in (7.15). After collecting coefficients of those terms that are defined in the current configuration, we obtain the following

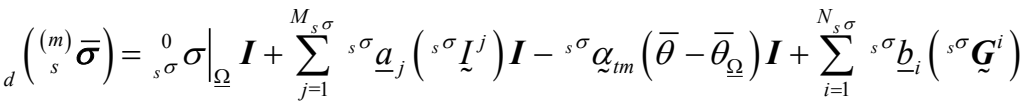

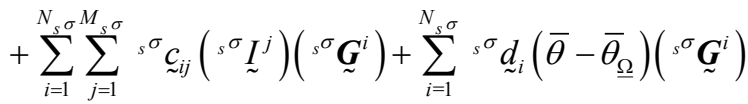

in which 


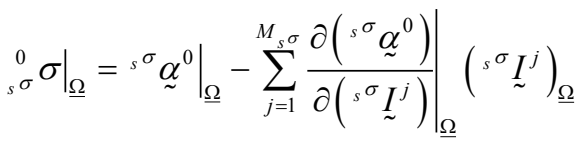

$$
\begin{aligned}
& { }_{s}^{\sigma} \underline{a}_{j}=\left.\frac{\partial\left({ }^{\sigma}{ }^{\sigma} \underline{\sim}^{0}\right)}{\partial\left({ }^{\sigma}{ }^{I_{\sim}^{j}}\right)}\right|_{\underline{\Omega}} ; j=1,2, \cdots, M_{s} \sigma \\
& { }^{\sigma} \underline{b}_{i}=\left.{ }^{\sigma}{ }_{\sim}^{\alpha}\right|_{\underline{\Omega}}-\left.\sum_{j=1}^{M_{s} \sigma} \frac{\partial\left({ }^{s} \sigma \underline{\sim}^{i}\right)}{\partial\left({ }^{\sigma} \sigma \underline{\sim}^{j}\right)}\right|_{\underline{\Omega}}\left({ }^{s}{ }_{\sim}{ }_{\sim}^{j}\right)_{\underline{\Omega}} ; i=1,2, \cdots, N_{s} \sigma \\
& { }^{\sigma}{ }_{\sim}{ }_{\sim j}=\left.\frac{\partial\left({ }^{\sigma}{ }^{\sigma} \underset{\sim}{\alpha}\right)}{\partial\left({ }^{\sigma}{ }^{\sigma} \underline{\sim}^{j}\right)}\right|_{\underline{\Omega}} ; j=1,2, \cdots, N_{s} \sigma \\
& { }_{s}^{\sigma}{\underset{\sim t m}{\alpha}}_{t m}=-\left.\frac{\partial\left({ }^{\sigma}{ }_{\underline{\sim}}^{\alpha}\right)}{\partial \bar{\theta}}\right|_{\underline{\Omega}} \\
& { }_{s} \sigma \underset{\sim}{d}=\left.\frac{\partial\left({ }_{s} \sigma \underset{\alpha^{i}}{\alpha}\right)}{\partial \bar{\theta}}\right|_{\underline{\Omega}} ; i=1,2, \cdots, N_{s} \sigma
\end{aligned}
$$

in which ${ }^{\sigma} \underline{a}_{j},{ }^{s} \underline{b}_{i},{ }^{s}{ }_{\sim} c_{i j},{ }^{\sigma} \sigma{\underset{\sim}{d}}_{i}$ and ${ }^{\sigma}{ }^{\sigma}{\underset{\sim}{t m}}_{\text {m }}$ are material coefficients defined in a known configuration $\underline{\Omega}$. This constitutive theory requires $\left(M_{s} \sigma+2 N_{s} \sigma+M_{s}{ } N_{s} \sigma+1\right)$ material coefficients. The material coefficients defined in (7.18) can be functions of $\left.\bar{\rho}\right|_{\underline{\Omega}},\left({ }^{\sigma}{ }_{\sim}^{I^{j}}\right)_{\Omega}$ and $\bar{\theta}_{\underline{\Omega}}$. This constitutive theory is based on integrity, the only approximation being truncation of the Taylor series expressions of ${ }^{s}{ }_{\sim}^{\alpha}{ }_{\sim}^{i} ; i=0,1, \cdots, N_{s} \sigma$. We consider simplified forms of this theory in later sections.

\subsection{Constitutive Theory for ${ }_{s}^{(0)} \bar{m}$}

We consider ${ }_{s}^{\left({ }^{1}\right)} \overline{\boldsymbol{m}}$ as constitutive variables. Its argument tensors are defined by (7.12). Similar to the derivation of the constitutive theory for the deviatoric part of the symmetric Cauchy stress, here also the constitutive theory should be a rate theory in time in the rates of symmetric moment tensor and symmetric part of the gradient tensor of the rotation rates. This is necessitated in order to incorporate physics of dissipation as well as memory due to the symmetric Cauchy moment tensor, its rates and the symmetric part of the gradient of rotation rate tensor. Let ${ }^{{ }^{m}} \boldsymbol{G}^{i} ; i=1,2, \cdots, N_{s}$ be the combined generators of the argument tensors of $\left.{ }^{\left({ }^{1} m\right.}\right)_{\bar{m}} \overline{\boldsymbol{m}}$ in (7.12) that are symmetric tensors of rank two and let ${ }^{m}{ }_{\sim}^{I^{j}} ; j=1,2, \cdots, M_{s}$ be the combined invariants of the same argument tensors, then using the representation theorem we can express ${ }_{{ }^{1} m}{ }_{s} \overline{\boldsymbol{m}}$ as a linear combination of ${ }^{m}{ }_{\sim} \boldsymbol{G}^{i} ; i=1,2, \cdots, N_{s}$ and $\boldsymbol{I}$ in the current configuration.

$$
{ }_{s}^{\left({ }^{1}\right)} \overline{\boldsymbol{m}}={ }^{s^{m}} \underset{\sim}{\alpha}{ }^{0} \boldsymbol{I}+\sum_{i=1}^{N_{s^{m}}}{ }^{s}{\underset{\sim}{\alpha}}^{i}\left({ }^{{ }^{m}}{\underset{\sim}{\boldsymbol{G}}}^{i}\right)
$$

in which

$$
{ }^{m}{\underset{\sim}{\alpha}}^{i}={ }^{m}{\underset{\sim}{\alpha}}^{i}\left(\bar{\rho},{ }^{m}{ }_{\sim}{ }_{\sim}^{j} ; j=1,2, \cdots, M_{s}, \bar{\theta}\right) ; i=0,1, \cdots, N_{s^{m}}
$$


To determine material coefficients in (7.19), we expand each ${ }^{m}{ }_{\sim}^{\alpha} ; i=0,1, \cdots, N_{s}$ in Taylor series in ${ }^{{ }^{m}}{ }_{\sim} I_{\sim}^{j} ; j=1,2, \cdots, M_{s^{m}}$ and $\bar{\theta}$ about a known configuration $\underline{\Omega}$, retaining only up to linear terms in ${ }^{m}{ }_{\sim}{ }^{j} ; j=1,2, \cdots, M_{s}$ and $\bar{\theta}$ (for simplicity) and then we substitute these ${ }^{m}{ }_{\sim}^{\alpha^{i}} ; i=0,1, \cdots, N_{s}$ in (7.19). After collecting coefficients of those terms that are defined in the current configuration, we obtain the following

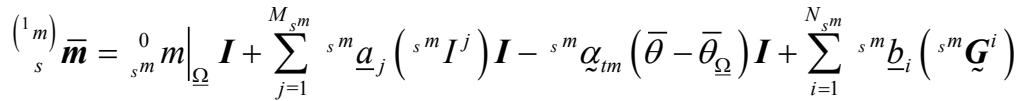

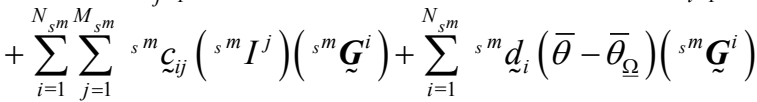

In which ${ }^{{ }^{m}} \underline{a}_{j},{ }^{m} \underline{b}_{i},{ }^{{ }^{m}}{ }_{\sim}{ }_{i j},{ }^{m}{ }_{\sim} \underset{i}{ }$ and ${ }^{s^{m}} \alpha_{t m}$ are material coefficients defined in the known configuration $\underline{\Omega}$. Explicit expressions for these can be obtained from (7.18) by replacing subscript and superscript ${ }_{s} \sigma$ with ${ }_{s} m$, hence the details are not repeated for the sake of brevity. This constitutive theory requires $\left(M_{s^{m}}+2 N_{s^{m}}+M_{s m} N_{s^{m}}+1\right)$ material coefficients. This constitutive theory is based on integrity. The only approximation being truncation of the Taylor series expansion of ${ }^{m}{\underset{\sim}{\alpha}}_{\sim}^{i} ; i=0,1, \cdots, N_{s m}$. Simplified forms of this constitutive theory are considered in later sections.

\subsection{Constitutive Theory for $\underset{a}{(0)} \bar{m}$}

Consider ${ }_{a}^{\left({ }^{m}\right)} \overline{\boldsymbol{m}}$ and its argument tensors defined by (7.13). ${ }_{a}^{\left({ }^{2}\right)} \overline{\boldsymbol{m}}$ is an antisymmetric tensor of rank two and so are its argument tensors except $\bar{\rho}$ and $\bar{\theta}$, these being tensors of rank zero. Let ${ }_{a^{m}}^{\boldsymbol{G}^{i}} ; i=1,2, \cdots, N_{a^{m}}$ be the combined generators of the argument tensors of ${ }_{a}^{\left({ }^{m}\right)} \overline{\boldsymbol{m}}$ that are antisymmetric tensors of rank two and let $a^{a^{m}}{\underset{\sim}{j}}^{j} ; j=1,2, \cdots, M_{a^{m}}$ be the combined invariants of the same argument tensors. Similar to Sections 7.3 and 7.4, in this case also we express ${ }_{a}^{\left({ }^{m}\right)} \overline{\boldsymbol{m}}$ as a linear combination of ${ }^{a^{m}} \boldsymbol{G}^{i} ; i=1,2, \cdots, N_{a}$ in the current configuration

$$
{ }_{a}^{\left({ }^{m}\right)} \overline{\boldsymbol{m}}=\sum_{i=1}^{N_{a^{m}}}{ }_{a^{m}}^{\alpha_{\sim}}{ }^{i}\left({ }^{{ }^{m}} \boldsymbol{G}^{i}\right)
$$

in which

$$
{ }^{a} \stackrel{\sim}{\alpha}^{i}={ }^{{ }^{m}}{\underset{\sim}{\alpha}}^{i}\left(\bar{\rho},{ }^{a^{m}}{\underset{\sim}{I}}^{j} ; j=1,2, \cdots, M_{a m}, \bar{\theta}\right) ; i=1,2, \cdots, N_{a^{m}}
$$

To determine material coefficients in (7.22), we expand each ${ }^{{ }^{m}}{\underset{\sim}{\alpha}}^{i} ; i=1,2, \cdots, N_{a^{m}}$ in Taylor series in ${ }^{a^{m}} \underset{\sim}{I^{j}} ; j=1,2, \cdots, M_{a^{m}}$ and $\bar{\theta}$ about a known configuration $\underline{\Omega}$, retaining only up to linear terms (for simplicity) in ${ }^{a^{m}} \underline{\sim}^{j} ; j=1,2, \cdots, M_{a^{m}}$ and $\bar{\theta}$ and then we substitute these ${ }^{a}{ }_{\sim} \alpha^{i} ; i=1,2, \cdots, N_{a^{m}}$ in (7.22). After collecting coefficients of those terms that are defined in the current configuration, we obtain the following

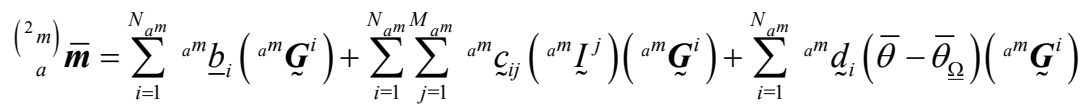


In which ${ }^{a^{m}} \underline{b}_{i},{ }^{a^{m}} c_{i j}$ and ${ }_{a^{m}} d_{i}$ are material coefficients defined in the known configuration $\underline{\Omega}$. Explicit expressions for these can be obtained from (7.18) by replacing subscript and superscript ${ }_{s} \sigma$ by ${ }_{a} m$. This constitutive theory requires $\left(2 N_{a^{m}}+M_{a^{m}} N_{a^{m}}\right)$ material coefficients. This constitutive theory is also based on integrity and has the same approximation as those in Sections 7.3 and 7.4. Simplified form of this constitutive theory will also be considered in later sections.

\subsection{Constitutive Theory for $\bar{q}$}

Recall inequality (50) resulting from the entropy inequality

$$
\overline{\boldsymbol{q}} \cdot \overline{\boldsymbol{g}} \leq 0 \quad(\text { as } \bar{\theta} \geq 0)
$$

In (7.25), $\overline{\boldsymbol{q}}$ and $\overline{\boldsymbol{g}}$ are conjugate. The simplest possible constitutive theory for $\overline{\boldsymbol{q}}$ can be derived by assuming that $\overline{\boldsymbol{q}}$ is proportional to $-\overline{\boldsymbol{g}}$ which leads to the following $\overline{\boldsymbol{q}}$ [38].

$$
\overline{\boldsymbol{q}}=-k(\bar{\theta}) \overline{\boldsymbol{g}}
$$

This is standard Fourier heat conduction law with temperature dependent thermal conductivity. Alternatively, if we assume (as in (6.9) after replacing $1 / \bar{\rho}$ by $\bar{\rho}$ )

$$
\overline{\boldsymbol{q}}=\overline{\boldsymbol{q}}(\bar{\rho}, \overline{\boldsymbol{g}}, \bar{\theta})
$$

then based on the representation theorem, we can begin with (as $\overline{\boldsymbol{g}}$ is the only combined generator of $\overline{\boldsymbol{g}}$ and $\bar{\theta}$ that is a tensor of rank one) the following in the current configuration

$$
\overline{\boldsymbol{q}}=-{ }^{q} \underset{\sim}{\alpha} \overline{\boldsymbol{g}}
$$

in which

$$
{ }^{q} \underset{\sim}{\alpha}={ }_{\sim}^{q} \underset{\sim}{\alpha}\left(\bar{\rho},{ }_{\sim}^{q}, \bar{\theta}\right) ;{ }_{\sim}^{q} \underset{\sim}{I} \overline{\boldsymbol{g}} \cdot \overline{\boldsymbol{g}}
$$

${ }^{q} \underset{\sim}{I}$ is the only invariant of the argument tensors of $\overline{\boldsymbol{g}}$ and $\bar{\theta}$. Expand ${ }^{q} \underset{\sim}{\alpha}$ in Taylor series in ${ }_{\sim}^{q} \underset{\sim}{I}$ and $\bar{\theta}$ about a known configuration $\underline{\Omega}$ and retaining only up to linear terms (for simplicity) in ${ }^{q} \underset{\sim}{I}$ and $\bar{\theta}$, we obtain the following [38] after collecting coefficients of the terms defined in the current configuration.

$$
\overline{\boldsymbol{q}}=-\left.k\right|_{\underline{\Omega}} \overline{\boldsymbol{g}}-\left.k_{1}\right|_{\underline{\underline{\Omega}}}(\overline{\boldsymbol{g}} \cdot \overline{\boldsymbol{g}}) \overline{\boldsymbol{g}}-\left.k_{2}\right|_{\underline{\underline{\Omega}}}\left(\bar{\theta}-\bar{\theta}_{\underline{\Omega}}\right) \overline{\boldsymbol{g}}
$$

where

$$
\begin{aligned}
& \left.k\right|_{\underline{\Omega}}=\left.{ }^{q} \underset{\sim}{\alpha}\right|_{\underline{\Omega}}+\left.\frac{\partial^{q} \underset{\sim}{\alpha}}{\partial\left({ }^{q} \underset{\sim}{I}\right)}\right|_{\underline{\underline{\Omega}}}\left(\{\bar{g}\}^{\mathrm{T}}\{\bar{g}\}\right)_{\underline{\Omega}} \\
& \left.k_{1}\right|_{\underline{\Omega}}=\left.\frac{\partial^{q} \underset{\sim}{\alpha}}{\partial\left({ }^{q} \underset{\sim}{I}\right)}\right|_{\underline{\Omega}} \\
& \left.k_{2}\right|_{\underline{\Omega}}=\left.\frac{\partial^{q} \underline{\alpha}}{\partial \bar{\theta}}\right|_{\underline{\Omega}}
\end{aligned}
$$


The constitutive theory (7.30) is the simplest possible constitutive theory based on representation theorem. $\left.k\right|_{\underline{\Omega}},\left.k_{1}\right|_{\underline{\Omega}}$ and $\left.k_{2}\right|_{\underline{\Omega}}$ can be functions of $\left.\bar{\rho}\right|_{\underline{\Omega}},\left.{ }^{q} I\right|_{\underline{\Omega}}$ and $\left.\bar{\theta}\right|_{\underline{\Omega}}$. Clearly the constitutive theory (7.26) is a subset of (7.30). This constitutive theory (7.30) is cubic in $\overline{\boldsymbol{g}}$.

\section{Simplified Constitutive Theories: Non-Classical and Classical Maxwell, Oldroyd-B, and Giesekus Constitutive Models}

In polymer science the Maxwell and the Oldroyd-B constitutive models derived using classical continuum mechanics (Surana et al. [40] and Surana [38]) are advocated [46] for dilute polymeric liquids that are dominantly viscous fluids with some elasticity whereas Giesekus constitutive model based in classical continuum mechanics (Surana et al. [41]) is advocated for dense polymeric fluids [46] in which the fluid behavior is elasticity dominated. The original derivations of these constitutive theories (see [46]) date back to the original papers by Maxwell, Oldroyd and Giesekus [46]. The derivations are explained using kinetic theory of gases, Brownian motion of polymer molecules, dumbbell models etc. Surana et al. [40] [41] and Surana [38] showed that these models in fact can be derived using principles of continuum mechanics, entropy inequality, the conditions resulting from the entropy inequality in conjunction with the representation theorem [38].

In references [38] [40] [41] authors derived ordered rate constitutive theories for polymeric fluids using convected time derivatives of the strain tensor up to order $n$ and the convected time derivatives of the Cauchy stress tensor of up to order $m$ based on classical continuum mechanics. They showed that 1) Maxwell model is a simplified linear constitutive model corresponding to $n=1$ and $m=1$; 2) Oldroyd-B model is a simplified quasilinear constitutive model corresponding to $n=2$ and $m=1$ that only contains Cauchy stress, its first convected time derivative and the first and second convected time derivatives of the strain tensor; 3) Giesekus model is same as Maxwell model but additionally contains quadratic term of the Cauchy stress tensor, thus this constitutive model is nonlinear. We make some remarks regarding the constitutive theories presented in Section 7 for non-classical compressible polymeric fluids.

\section{Remarks}

1) The ordered rate constitutive theories presented here for non-classical polymeric fluids naturally contains the ordered rate constitutive theories for the classical polymeric fluids as subset. These are easily obtained by removing the internal rotation rate physics that requires ${ }_{s}^{(0)} \overline{\boldsymbol{m}}={ }_{a}^{(0)} \overline{\boldsymbol{m}}=0$ and the Cauchy stress tensor to be symmetric due to balance of angular momenta. The resulting constitutive theory is same as in references [38] [40] [41] for classical polymeric fluids.

2) Since the constitutive theories presented here are based on integrity, all specific simplified forms of the constitutive models are all subset of these. Hence, it should be possible to present a single non-classical constitutive model for di- 
lute as well as dense polymeric fluids which would also contain commonly used current constitutive models (based on classical mechanics) within a single constitutive theory. In order for the non-classical constitutive theories to contain currently used classical constitutive theories we need to choose the following for the ordered rates. $n=2, m=1,{ }^{1} m=1,{ }^{2} m=1$. For this choice the constitutive variables and their argument tensors are

$$
\begin{aligned}
& \left.{ }_{d}^{\left({ }_{s}\right)} \overline{\boldsymbol{\sigma}}\right)={ }_{d}\left({ }_{s}^{(1)} \overline{\boldsymbol{\sigma}}\right)\left(\bar{\rho},{ }^{(1)} \boldsymbol{\gamma},{ }^{(2)} \gamma,{ }_{d}\left({ }_{s}^{(0)} \overline{\boldsymbol{\sigma}}\right), \bar{\theta}\right) \\
& { }_{s}^{(1)} \overline{\boldsymbol{m}}={ }_{s}^{(1)} \overline{\boldsymbol{m}}\left(\bar{\rho},{ }_{s}^{t} \boldsymbol{J},{ }_{s}^{(0)} \overline{\boldsymbol{m}}, \bar{\theta}\right) \\
& { }_{a}^{(1)} \overline{\boldsymbol{m}}={ }_{a}^{(1)} \overline{\boldsymbol{m}}\left(\bar{\rho},{ }_{a}^{t} \boldsymbol{J},{ }_{a}^{(0)} \overline{\boldsymbol{m}}, \bar{\theta}\right)
\end{aligned}
$$

Constitutive theories derived using (8.1) when based on integrity will require too many material coefficients for deviatoric part of the symmetric Cauchy stress tensor as well as for each of the moment tensors. Constitutive theory for $\overline{\boldsymbol{q}}$ remains unaffected. We consider the following simplifications

1) Consider the constitutive theories to be linear in ${ }^{(1)} \gamma,{ }^{(2)} \gamma,{ }_{s}^{t} \Theta \boldsymbol{J}$ and ${ }_{a}^{t} \Theta \boldsymbol{J}$.

2) Neglect the product terms of ${ }^{(1)} \gamma,{ }^{(2)} \gamma,{ }_{s}^{t}{ }_{s} \boldsymbol{J},{ }_{a}^{t} \boldsymbol{J},\left({ }_{s}^{(0)} \overline{\boldsymbol{\sigma}}\right),{ }_{s}^{(0)} \overline{\boldsymbol{m}}$, and ${ }_{a}^{(0)} \overline{\boldsymbol{m}}$.

3) Neglect all $\left(\bar{\theta}-\left.\bar{\theta}\right|_{\underline{\Omega}}\right)$ terms (to conform to the currently used constitutive models).

4) Also neglect the first term in each constitutive theory containing influence of initial stress and initial moments.

5) We consider generators $\left({ }_{d}\left({ }_{s}^{(0)} \overline{\boldsymbol{\sigma}}\right)\right)^{2},\left({ }_{s}^{(0)} \overline{\boldsymbol{m}}\right)^{2}$, and $\left({ }_{a}^{(0)} \overline{\boldsymbol{m}}\right)^{2}$ but neglect quadratic and cubic trace terms in the invariants as well as products of these generators with others.

Based on these assumptions we obtain the following constitutive theories for stress and moment tensors.

\subsection{Constitutive Theory for Deviatoric Part of the Symmetric Cauchy Stress Tensor}

If we consider

$$
\begin{aligned}
& { }^{{ }^{\sigma}} \boldsymbol{G}^{1}={ }^{(1)} \boldsymbol{\gamma}, \quad{ }^{{ }^{\sigma}} \boldsymbol{G}^{2}={ }^{(2)} \boldsymbol{\gamma}, \quad{ }^{{ }^{\sigma}} \boldsymbol{G}^{3}={ }_{d}\left({ }_{s}^{(0)} \overline{\boldsymbol{\sigma}}\right), \quad{ }^{\circ} \boldsymbol{G}^{4}=\left({ }_{d}\left({ }_{s}^{(0)} \overline{\boldsymbol{\sigma}}\right)\right)^{2},
\end{aligned}
$$

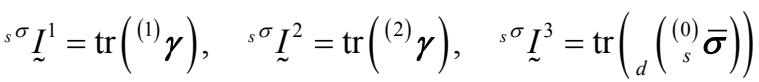

then we obtain the following from (7.17) (using $m=1, n=2$ ) based on restrictions 1) - 5).

$$
\begin{aligned}
& { }_{d}\left({ }_{s}^{(1)} \overline{\boldsymbol{\sigma}}\right)={ }^{s} \underline{a}_{1}\left(\operatorname{tr}\left({ }^{(1)} \boldsymbol{\gamma}\right)\right) \boldsymbol{I}+{ }^{\sigma}{ }^{\sigma} \underline{a}_{2}\left(\operatorname{tr}\left({ }^{(2)} \boldsymbol{\gamma}\right)\right) \boldsymbol{I}+{ }^{\sigma}{ }^{\sigma} \underline{a}_{3}\left(\operatorname{tr}\left({ }_{d}\left({ }_{s}^{(0)} \overline{\boldsymbol{\sigma}}\right)\right)\right) \boldsymbol{I} \\
& +{ }^{\circ} \underline{b}_{1}\left({ }^{(1)} \gamma\right)+{ }^{\sigma} \underline{b}_{2}\left({ }^{(2)} \gamma\right)+{ }^{\sigma} \underline{b}_{3}\left({ }_{d}\left({ }_{s}^{(0)} \bar{\sigma}\right)\right)+{ }^{\sigma}{ }^{\sigma} \underline{b}_{4}\left({ }_{d}\left({ }_{s}^{(0)} \bar{\sigma}\right)\right)^{2}
\end{aligned}
$$

In order to rewrite (8.3) in standard easily recognizable form, we transfer ${ }^{5}{ }_{b_{3}}\left({ }_{d}\left({ }_{s}^{(0)} \bar{\sigma}\right)\right)$ term to the left side of (8.3) and divide the entire equation by ${ }_{-s}{ }^{\sigma} \underline{b}_{3}$ and define new coefficients as follows. 


$$
\begin{aligned}
& \lambda=\left(-\frac{1}{{ }^{s} \underline{b}_{3}}\right), \quad 2 \eta=\left(-\frac{{ }^{s}{ }^{\sigma} \underline{b}_{1}}{{ }^{\sigma} \sigma \underline{b}_{3}}\right), \quad \kappa=\left(-\frac{{ }^{s}{ }^{\sigma} \underline{a}_{1}}{{ }^{\sigma} \sigma \underline{b}_{3}}\right) \\
& { }^{1} \kappa=\left(-\frac{{ }^{s} \sigma \underline{a}_{3}}{{ }^{\sigma} \underline{b}_{3}}\right), \quad{ }^{2} \kappa=\left(-\frac{{ }^{s} \underline{a}_{2}}{{ }^{\sigma} \underline{b}_{3}}\right), \quad 2 \eta \lambda_{2}=\left(-\frac{{ }^{s} \underline{b}_{2}}{{ }^{\sigma} \underline{b}_{3}}\right), \quad \frac{\lambda}{\eta} \alpha=\left(-\frac{{ }^{s} \underline{b}_{4}}{{ }^{\sigma} \underline{b}_{3}}\right)
\end{aligned}
$$

then (8.3) can be written as

$$
\begin{aligned}
{ }_{d}\left({ }_{s}^{(0)} \overline{\boldsymbol{\sigma}}\right)+\lambda\left({ }_{d}\left({ }_{s}^{(1)} \overline{\boldsymbol{\sigma}}\right)\right)= & 2 \eta\left({ }^{(1)} \boldsymbol{\gamma}\right)+2 \eta \lambda_{2}\left({ }^{(2)} \boldsymbol{\gamma}\right)+\frac{\lambda}{\eta} \alpha\left({ }_{d}\left({ }_{s}^{(0)} \overline{\boldsymbol{\sigma}}\right)\right)^{2} \\
& \left.+\kappa\left(\operatorname{tr}\left({ }^{(1)} \boldsymbol{\gamma}\right)\right) \boldsymbol{I}+{ }^{1} \kappa\left(\operatorname{tr}\left({ }_{d}\left({ }^{(0)} \overline{\boldsymbol{\sigma}}\right)\right)\right)\right) \boldsymbol{I}+{ }^{2} \kappa\left(\operatorname{tr}\left({ }^{(2)} \boldsymbol{\gamma}\right)\right) \boldsymbol{I}
\end{aligned}
$$

In which $\eta$ is viscosity, $\lambda$ is relaxation time, $\lambda_{2}$ is retardation time, $\kappa$ and ${ }^{2} \kappa$ are second viscosity and the viscosity associated with ${ }^{(2)} \gamma$, and $\alpha$ is mobility factor. This constitutive model holds for compressible polymeric fluids. In case of incompressible fluids, $\operatorname{tr}\left[{ }^{(1)} \gamma\right]=\operatorname{tr}[\bar{D}]=0$ can be used to simplify (8.5).

\section{Maxwell model: Compressible}

To obtain Maxwell model from (8.5) we set ${ }^{(2)} \gamma=0, \alpha=0,{ }^{1} \kappa=0$ and ${ }^{2} \kappa=0$. The resulting constitutive theory is given by

$$
{ }_{d}\left({ }_{s}^{(0)} \overline{\boldsymbol{\sigma}}\right)+\lambda\left({ }_{d}\left({ }_{s}^{(1)} \overline{\boldsymbol{\sigma}}\right)\right)=2 \eta\left({ }^{(1)} \boldsymbol{\gamma}\right)+\kappa\left(\operatorname{tr}\left({ }^{(1)} \boldsymbol{\gamma}\right)\right) \boldsymbol{I}
$$

This is a linear viscoelastic Maxwell model.

\section{Oldroyd-B model: Compressible}

To derive this constitutive model we also use (8.5) with $\alpha=0,{ }^{1} \kappa=0$ and ${ }^{2} \kappa=0$ to obtain a quasilinear viscoelastic model (nonlinearity due to ${ }^{(2)} \gamma$ ).

$$
{ }_{d}\left({ }_{s}^{(0)} \overline{\boldsymbol{\sigma}}\right)+\lambda\left({ }_{d}\left({ }^{(1)} \overline{\boldsymbol{\sigma}}\right)\right)=2 \eta\left({ }^{(1)} \boldsymbol{\gamma}\right)+2 \eta \lambda_{2}\left({ }^{(2)} \boldsymbol{\gamma}\right)+\kappa\left(\operatorname{tr}\left({ }^{(1)} \boldsymbol{\gamma}\right)\right) \boldsymbol{I}
$$

\section{Giesekus model: Compressible}

In this constitutive model we use (8.5) and set $\lambda_{2}=0,{ }^{1} \kappa=0$ and ${ }^{2} \kappa=0$ to obtain the Giesekus constitutive model.

$$
{ }_{d}\left({ }_{s}^{(0)} \overline{\boldsymbol{\sigma}}\right)+\lambda\left({ }_{d}\left({ }_{s}^{(1)} \overline{\boldsymbol{\sigma}}\right)\right)=2 \eta\left({ }^{(1)} \boldsymbol{\gamma}\right)+\kappa\left(\operatorname{tr}\left({ }^{(1)} \boldsymbol{\gamma}\right)\right) \boldsymbol{I}+\frac{\lambda}{\eta} \alpha\left({ }_{d}\left({ }_{s}^{(0)} \overline{\boldsymbol{\sigma}}\right)\right)^{2}
$$

\section{Remarks}

1) The constitutive models (8.5)-(8.8) are valid in case of non-classical as well as classical continuum theories.

2) The constitutive theory (8.5) is valid for Maxwell model, Oldroyd-B model as well as Giesekus model based on classical continuum theory with appropriate choice of material coefficients. Thus, there is no need for (8.6)-(8.8). When calibrating (8.5), for dilute or dense polymeric fluids the material coefficients that are not applicable for the physics under consideration will automatically assume zero or small values.

3) In case of constitutive theories for non-classical continuum mechanics in addition to (8.5) we also need constitutive theory(ies) for the moment ten$\operatorname{sor}(\mathrm{s})$. 


\subsection{Constitutive Theory for ${ }^{(0)} \bar{m}$}

Consider dependent variable ${ }_{s}^{(1)} \overline{\boldsymbol{m}}$ and its argument tensors in (8.1). As mentioned earlier, the constitutive theory based on integrity will require too many material coefficients. If we consider

$$
\begin{aligned}
& { }^{{ }^{m}} \boldsymbol{G}^{1}={ }_{s}^{t} \boldsymbol{J}, \quad{ }^{m} \boldsymbol{G}^{2}={ }_{s}^{(0)} \overline{\boldsymbol{m}}, \quad{ }^{m} \boldsymbol{G}^{3}=\left({ }_{s}^{(0)} \overline{\boldsymbol{m}}\right)^{2} \\
& { }^{m}{ }_{\sim} I^{1}=\operatorname{tr}\left({ }_{i}^{t} \boldsymbol{J}\right), \quad{ }^{m}{ }_{\sim} I^{2}=\operatorname{tr}\left({ }_{s}^{(0)} \overline{\boldsymbol{m}}\right)
\end{aligned}
$$

then we can obtain the following from (7.21) (based on assumptions 1) - 5) stated in Section 8).

$$
\begin{aligned}
{ }_{s}^{(1)} \overline{\boldsymbol{m}}= & { }^{s} \underline{a}_{1}\left(\operatorname{tr}\left({ }_{i s}^{t} \Theta \boldsymbol{J}\right)\right) \boldsymbol{I}+{ }^{m} \underline{a}_{2}\left(\operatorname{tr}\left({ }_{s}^{(0)} \overline{\boldsymbol{m}}\right)\right) \boldsymbol{I}+{ }^{m} \underline{b}_{1}\left(\begin{array}{c}
{ }_{i}^{t} \Theta \\
s
\end{array}\right) \\
& +{ }^{m} \underline{b}_{2}\left({ }_{s}^{(0)} \overline{\boldsymbol{m}}\right)+{ }^{m} \underline{b}_{3}\left({ }_{s}^{(0)} \overline{\boldsymbol{m}}\right)^{2}
\end{aligned}
$$

We transfer ${ }^{m} \underline{b}_{2}\left({ }_{s}^{(0)} \overline{\boldsymbol{m}}\right)$ term to the left side in (8.10) and divide the entire equation by $\left(-s^{m} \underline{b}_{2}\right)$ and define

$$
\begin{aligned}
& \left.{ }^{m} \lambda=\left(-\frac{1}{{ }^{s} \underline{b}_{2}}\right), \quad 2\left({ }^{m} \eta\right)=\left(-{ }^{{ }^{m} \underline{b}_{1}}\right), \quad{ }^{{ }^{m} \underline{b}_{2}}\right), \quad{ }^{m} \kappa=\left(-\frac{{ }^{s} \underline{a}_{1}}{{ }^{m} \underline{b}_{2}}\right) \\
& \frac{{ }^{m} \lambda}{{ }^{m} \eta} \eta\left({ }^{m} \alpha\right)=\left(-\frac{{ }^{s} \underline{b}_{3}}{{ }^{m} \underline{b}_{2}}\right), \quad{ }^{m}\left({ }^{1} \kappa\right)=\left(-\frac{{ }^{s} \underline{a}_{2}}{{ }^{m} \underline{b}_{2}}\right)
\end{aligned}
$$

then, (8.10) can be written as

$$
\begin{aligned}
& { }_{s}^{(0)} \overline{\boldsymbol{m}}+{ }^{m} \lambda\left({ }^{(1)} \overline{\boldsymbol{m}}\right)=2\left({ }^{m} \eta\right)\left({ }_{i}^{t} \Theta \boldsymbol{J}\right)+{ }^{m} \kappa\left(\operatorname{tr}\left({ }_{i}^{t} \Theta \boldsymbol{J}\right)\right) \boldsymbol{I} \\
& +{ }^{m}\left({ }^{1} \kappa\right)\left(\operatorname{tr}\left({ }^{(0)} \overline{\boldsymbol{m}}\right)\right) \boldsymbol{I}+\frac{{ }^{m}{ }^{m} \lambda}{{ }^{m} \eta}\left(s^{m} \alpha\right)\left({ }_{s}^{(0)} \overline{\boldsymbol{m}}\right)^{2}
\end{aligned}
$$

\section{Maxwell and Oldroyd-B models}

Following the derivation of constitutive theory for deviatoric symmetric Cauchy stress tensor, in (8.12) if we set $s^{m}\left({ }^{1} \kappa\right)=0$ and ${ }^{m} \alpha=0$, then we obtain

$$
{ }_{s}^{(0)} \overline{\boldsymbol{m}}+{ }^{m} \lambda\left({ }_{s}^{(1)} \overline{\boldsymbol{m}}\right)=2\left({ }^{m} \eta\right)\left({ }_{i}^{t} \Theta \boldsymbol{J}\right)+{ }^{m} \boldsymbol{\kappa} \kappa\left(\operatorname{tr}\left({ }_{s}^{t} \Theta \boldsymbol{J}\right)\right) \boldsymbol{I}
$$

\section{Giesekus model}

Consistent with the derivation of constitutive model for classical continuum theory, we set $s^{m}\left({ }^{1} \kappa\right)=0$ to obtain the following constitutive model from (8.12).

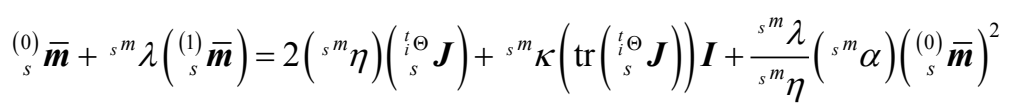

\section{Remarks}

1) It is rather obvious that (8.13) and (8.14) are a subset of (8.12). When calibrating (8.12), the material coefficients not contributing to the physics will automatically be zero (or small values). 
2) Thus, we see that for symmetric Cauchy moment tensor also we need to consider only a single constitutive model (8.12).

\subsection{Constitutive Theory for ${ }_{a}^{(0)} \bar{m}$}

Consider the constitutive variable ${ }_{a}^{(1)} \overline{\boldsymbol{m}}$ and its argument tensors in (8.1). Since ${ }_{a}^{(0)} \overline{\boldsymbol{m}}$ and two of its four argument tensors are antisymmetric tensors of rank two, the combined generators of the argument tensors and invariants are quite limited. Thus, for this constitutive theory we consider integrity first before any simplification. Based on (8.1) we have the following combined generators and invariants

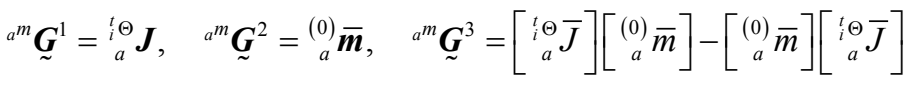

and

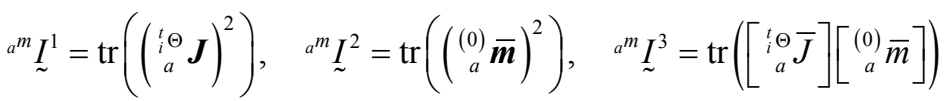

This constitutive theory will naturally lead to a large number of material coefficients $\left(N_{a^{m}}=3, M_{a^{m}}=3\right)$. The choice of which generators and invariants to retain is not simple as neglecting nonlinear and product terms in this case is quite detrimental. For illustrative purposes we consider a constitutive theory that is linear in ${ }_{a}^{t} \Theta \boldsymbol{J}$ and ${ }_{a}^{(0)} \overline{\boldsymbol{m}}$ and contains the product of these terms as well, i.e., we consider all three generators but only invariant ${ }^{a^{m}}{\underset{\sim}{3}}^{3}$. In this theory products of the generators and the invariant ${ }^{a^{m}} I_{\sim}^{3}$ is not admissible as this would contain a quadratic term in ${ }_{a}^{t} \Theta \boldsymbol{J}$ and ${ }_{a}^{(0)} \overline{\boldsymbol{m}}$. Thus we can write (following (8.10), neglecting $\left(\bar{\theta}-\left.\bar{\theta}\right|_{\underline{\Omega}}\right)$ terms)

$$
{ }_{a}^{(1)} \overline{\boldsymbol{m}}={ }^{a} \underline{b}_{1}\left({ }_{i}^{t} \Theta \boldsymbol{J}\right)+{ }^{a^{m}} \underline{b}_{2}\left({ }_{a}^{(0)} \overline{\boldsymbol{m}}\right)+{ }^{a^{m}} \underline{b}_{3}\left(\left({ }_{i}^{t} \Theta \boldsymbol{J}\right)(\underset{a}{(0)} \overline{\boldsymbol{m}})-(\underset{a}{(0)} \overline{\boldsymbol{m}})\left({ }_{i}^{t} \Theta \boldsymbol{J}\right)\right)
$$

transferring ${ }^{a^{m}} \underline{b}_{2}\left({ }_{a}^{(0)} \overline{\boldsymbol{m}}\right)$ to the left side of (8.17) and dividing the whole equation by $-a^{a^{m}} \underline{b}_{2}$ and defining

$$
{ }^{{ }^{m}} \lambda=-\frac{1}{{ }^{a^{m}} \underline{b}_{2}} ; \quad 2\left({ }^{a}{ }^{m} \eta_{1}\right)=\left(-\frac{{ }^{a^{m}} \underline{b}_{1}}{{ }^{a^{m}} \underline{b}_{2}}\right) ; \quad{ }^{a}{ }^{m} \eta_{2}=\left(-\frac{{ }^{a^{m}} \underline{b}_{3}}{{ }^{a^{m}} \underline{b}_{2}}\right)
$$

we obtain

$$
{ }_{a}^{(0)} \overline{\boldsymbol{m}}+{ }^{a}{ }^{m} \lambda\left({ }_{a}^{(1)} \overline{\boldsymbol{m}}\right)=2\left({ }^{a} \eta_{1}\right)\left({ }_{a}^{t} \Theta \boldsymbol{J}\right)+{ }^{a}{ }^{m} \eta_{2}\left(\left({ }_{i}^{t} \Theta \boldsymbol{J}\right)(\underset{a}{(0)} \overline{\boldsymbol{m}})-\left({ }_{a}^{(0)} \overline{\boldsymbol{m}}\right)\left({ }_{i}^{t} \Theta \boldsymbol{J}\right)\right)
$$

The constitutive theory (8.19) is linear in ${ }_{a}^{t} \Theta \boldsymbol{J}$ and ${ }_{a}^{(0)} \overline{\boldsymbol{m}}$ but contains their product terms.

\section{Maxwell model}

This following constitutive model, similar to Maxwell model for classical mechanics, is easily deduced from (8.19) by setting ${ }^{a} \eta_{2}=0$.

$$
{ }_{a}^{(0)} \overline{\boldsymbol{m}}+{ }^{a}{ }^{m} \lambda\left({ }_{a}^{(1)} \overline{\boldsymbol{m}}\right)=2\left({ }^{a}{ }^{m} \eta_{1}\right)\left({ }_{i}^{{ }_{i} \Theta} \boldsymbol{J}\right)
$$

\section{Oldroyd-B and Giesekus models}

Derivation of these models (parallel to those based on classical continuum 
mechanics) from (8.19) is not clear as this model (8.19) does not have second convected time derivative of the rotation gradient tensor (needed for Oldroyd-B model) and $\left({ }_{a}^{(0)} \overline{\boldsymbol{m}}\right)^{2}$ term needed for Giesekus model. Even the constitutive model based on integrity does not contain these terms. We simply leave with (8.19) at this stage.

\section{Complete Mathematical Model}

In the following we provide complete mathematical model including the constitutive theories for non-classical thermoviscoelastic fluids in simplified forms that contain the constitutive theories based on classical continuum theories as subset. The constitutive theories for classical case are easily obtained by eliminating the moment tensor and the constitutive theories for it and recognizing that for this case the Cauchy stress tensor is symmetric. In the following we assume that the balance of moment of moments is not a balance law, hence Cauchy moment tensor is not symmetric.

Conservation and balance laws

$$
\begin{aligned}
& \frac{\partial \bar{\rho}}{\partial t}+\bar{\nabla} \cdot(\bar{\rho} \overline{\boldsymbol{v}})=0 \\
& \bar{\rho} \frac{\partial \overline{\boldsymbol{v}}}{\partial t}+\bar{\rho}_{j} \frac{\partial \bar{v}_{i}}{\partial \bar{x}_{j}}-\bar{\rho} \overline{\boldsymbol{F}}_{i}^{b}-\frac{\partial}{\partial \bar{x}_{j}}\left({ }^{(0)} \bar{\sigma}_{j i}\right)=0 \\
& { }^{(0)} \bar{m}_{p k, p}-\epsilon_{i j k}{ }^{(0)} \bar{\sigma}_{i j}=0
\end{aligned}
$$

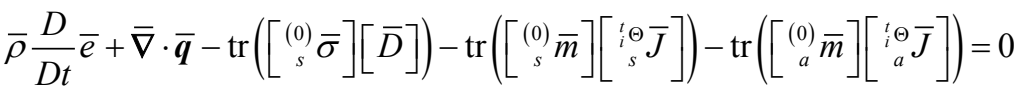

$$
\begin{aligned}
& \frac{\overline{\boldsymbol{q}} \cdot \overline{\boldsymbol{g}}}{\bar{\theta}}-\operatorname{tr}\left(\left[{ }_{s}^{(0)} \bar{\sigma}\right][\bar{D}]\right)-\operatorname{tr}\left(\left[{ }^{(0)} \bar{m}\right]\left[\left[{ }_{i}^{\dagger} \bar{s} \bar{J}\right]\right)-\operatorname{tr}\left(\left[{ }^{(0)} \bar{m}\right]\left[\left[{ }_{i}^{t}{ }_{a} \bar{J}\right]\right) \leq 0\right.\right. \\
& { }^{(0)} \overline{\boldsymbol{\sigma}}={ }_{s}^{(0)} \overline{\boldsymbol{\sigma}}+{ }_{a}^{(0)} \overline{\boldsymbol{\sigma}} ; \quad{ }_{s}^{(0)} \overline{\boldsymbol{\sigma}}={ }_{e}\left({ }_{s}^{(0)} \overline{\boldsymbol{\sigma}}\right)+{ }_{d}\left({ }_{s}^{(0)} \overline{\boldsymbol{\sigma}}\right) ; \quad{ }^{m} \underline{b}_{i}={ }_{s}^{(0)} \overline{\boldsymbol{m}}+{ }_{a}^{(0)} \overline{\boldsymbol{m}}
\end{aligned}
$$

Constitutive theories using general constitutive theories (8.5), (8.12), (8.19), and (7.30) applicable to dilute as well as dense polymers

$$
\begin{aligned}
& e^{\left({ }_{s}^{(0)} \overline{\boldsymbol{\sigma}}\right)}= \begin{cases}\bar{p}(\bar{\rho}, \bar{\theta}) \boldsymbol{I} ; & \text { compressible } \\
\bar{p}(\bar{\theta}) \boldsymbol{I} ; & \text { incompressible }\end{cases} \\
& { }_{d}\left({ }_{s}^{(0)} \bar{\sigma}\right)+\lambda\left({ }_{d}\left({ }_{s}^{(1)} \bar{\sigma}\right)\right) \\
& =2 \eta\left({ }^{(1)} \gamma\right)+2 \eta \lambda_{2}\left({ }^{(2)} \gamma\right)+\frac{\lambda}{\eta} \alpha\left({ }_{d}\left({ }_{s}^{(0)} \bar{\sigma}\right)\right)^{2} \\
& +\kappa\left(\operatorname{tr}\left({ }^{(1)} \gamma\right)\right) \boldsymbol{I}+{ }^{1} \kappa\left(\operatorname{tr}\left({ }_{d}\left({ }_{s}^{(0)} \overline{\boldsymbol{\sigma}}\right)\right)\right) \boldsymbol{I}+{ }^{2} \kappa\left(\operatorname{tr}\left({ }^{(2)} \gamma\right)\right) \boldsymbol{I} \\
& { }_{s}^{(0)} \overline{\boldsymbol{m}}+{ }^{m} \lambda\left({ }_{s}^{(1)} \overline{\boldsymbol{m}}\right)=2\left({ }^{m} \eta\right)\left({ }_{i}^{t} \Theta \boldsymbol{J}\right)+{ }^{m} \kappa\left(\operatorname{tr}\left({ }_{i}^{t} \Theta \boldsymbol{J}\right)\right) \boldsymbol{I} \\
& +{ }^{m}\left({ }^{1} \kappa\right)\left(\operatorname{tr}\left({ }_{s}^{(0)} \overline{\boldsymbol{m}}\right)\right) \boldsymbol{I}+\frac{{ }^{s} m}{{ }^{m} m} \eta\left({ }^{m} \alpha\right)\left({ }_{s}^{(0)} \overline{\boldsymbol{m}}\right)^{2}
\end{aligned}
$$




$$
\begin{aligned}
& { }_{a}^{(0)} \overline{\boldsymbol{m}}+{ }^{a m} \lambda\left({ }_{a}^{(1)} \overline{\boldsymbol{m}}\right)=2\left({ }^{a} \eta_{1}\right)\left({ }_{a}^{t} \Theta \boldsymbol{J}\right)+{ }^{a} m \eta_{2}\left(\left(\begin{array}{c}
{ }_{i}^{t} \Theta \\
a
\end{array}\right)\left(\underset{a}{{ }_{a}} \overline{\boldsymbol{m}}\right)-\left({ }_{a}^{(0)} \overline{\boldsymbol{m}}\right)\left({ }_{i}^{t} \Theta \boldsymbol{J}\right)\right) \\
& \overline{\boldsymbol{q}}=-\left.k\right|_{\underline{\Omega}} \overline{\boldsymbol{g}}-\left.k_{1}\right|_{\underline{\Omega}}(\overline{\boldsymbol{g}} \cdot \overline{\boldsymbol{g}}) \overline{\boldsymbol{g}}-\left.k_{2}\right|_{\underline{\Omega}}\left(\bar{\theta}-\bar{\theta}_{\underline{\Omega}}\right) \overline{\boldsymbol{g}}
\end{aligned}
$$

We show that this mathematical model has closure for compressible as well as incompressible non-classical polymeric fluids.

\section{Compressible:}

For this case thermodynamic pressure $\bar{p}(\bar{\rho}, \bar{\theta})$ is defined by an equation of state and $\bar{e}=\bar{e}(\bar{p}, \bar{\rho}, \bar{\theta})=\bar{e}(\bar{\rho}, \bar{\theta})$ is also known, hence $\bar{p}$ and $\bar{e}$ are not dependent variables in the mathematical model. Thus we have (number in the brackets is the count of the number of variables):

$\left.\bar{\rho}(1), \overline{\boldsymbol{v}}(3),{ }_{d}{ }_{s}^{(0)} \overline{\boldsymbol{\sigma}}\right)(6),{ }_{a}^{(0)} \overline{\boldsymbol{\sigma}}(3), \overline{\boldsymbol{q}}(3), \bar{\theta}(1),{ }_{s}^{(0)} \overline{\boldsymbol{m}}(6),{ }_{a}^{(0)} \overline{\boldsymbol{m}}(3)$, a total of 26 dependent variables. The number of equations in this mathematical model are: continuity (1), balance of linear momenta (3), balance of angular momenta (3), energy equation (1), constitutive theories for:

$\left.{ }_{d}^{\left({ }^{(0)}\right.} \overline{\boldsymbol{\sigma}}\right)(6),{ }_{s}^{(0)} \overline{\boldsymbol{m}}(6),{ }_{a}^{(0)} \overline{\boldsymbol{m}}(3), \overline{\boldsymbol{q}}(3)$, a total of 26 , hence this mathematical model has closure.

\section{Incompressible:}

In this case $\bar{\rho}=\rho_{0}=$ constant, hence known, but the pressure $\bar{p}=\bar{p}(\bar{\theta})$ is not known, hence the number of equations as well as the number of variables for this case also remains 26 but instead of known $\bar{p}(\bar{\rho}, \bar{\theta}), \bar{p}(\bar{\theta})$ becomes an unknown dependent variable.

\section{Remarks}

1) When balance of moment of moments is considered as a balance law [75] [76] [77], then Cauchy moment tensor ${ }^{(0)} \overline{\boldsymbol{m}}$ becomes symmetric, i.e., ${ }_{s}^{(0)} \overline{\boldsymbol{m}}={ }^{(0)} \overline{\boldsymbol{m}}$ and ${ }_{a}^{(0)} \overline{\boldsymbol{m}}=0$. This eliminates ${ }_{a}^{(0)} \overline{\boldsymbol{m}}$, three as dependent variables in the mathematical model as well as three constitutive equations for ${ }_{a}^{(0)} \overline{\boldsymbol{m}}$. Thus, for this case we have 23 dependent variables and 23 equations.

2) The mathematical model uses basis independent measures, i.e.,

$\left({ }_{s}^{(0)} \overline{\boldsymbol{\sigma}}\right),{ }_{a}^{(0)} \overline{\boldsymbol{\sigma}},{ }_{s}^{(0)} \overline{\boldsymbol{m}},{ }_{a}^{(0)} \overline{\boldsymbol{m}}, \cdots$, etc . This mathematical model can easily be made basis dependent by choosing these measures in contravariant basis or covariant basis or using Jaumann measure keeping in mind that if these measures are contravariant then the corresponding conjugate quantities are covariant or vice-versa.

3) These polymeric fluids have elasticity, dissipation mechanism, as well as memory. Elasticity is due to stretching of long chain polymer molecules, dissipation is due to both short chain molecules of solvent as well as long chain molecules of polymer and their interactions and memory is due to relaxation phenomenon inherent in these fluids because of stretched polymer molecules resuming their unstressed (or relaxed state).

4) Dissipation and memory mechanism in these non-classical polymeric fluids are due to ${ }_{d}\left({ }_{s}^{(0)} \overline{\boldsymbol{\sigma}}\right),{ }_{s}^{(0)} \overline{\boldsymbol{m}}$ as well as ${ }_{a}^{(0)} \overline{\boldsymbol{m}}$ (when balance of moment of moments is not used as a balance law). These are fully accounted for in the constitutive theories based on integrity as well as their simplified general forms ((9.8), 
(9.9), and (9.10)) and their specific forms that are only valid for Maxwell, Oldroyd-B, and Giesekus constitutive models respectively.

\section{Retardation and Memory Moduli}

Using (9.8)-(9.10) and discarding $\left.{ }_{d}^{(}{ }_{s}^{(0)} \overline{\boldsymbol{\sigma}}\right),{ }_{s}^{(0)} \overline{\boldsymbol{m}}$ and ${ }_{a}^{(0)} \overline{\boldsymbol{m}}$ terms on the right hand sides and defining ${ }^{\sigma} \boldsymbol{Q}_{s},{ }^{m} \boldsymbol{Q}_{s}$ and ${ }^{m} \boldsymbol{Q}_{a}$

$$
\begin{gathered}
{ }^{\sigma} \boldsymbol{Q}_{s}=2 \eta\left({ }^{(1)} \boldsymbol{\gamma}\right)+2 \eta \lambda_{2}\left({ }^{(2)} \gamma\right)+\kappa\left(\operatorname{tr}\left({ }^{(1)} \boldsymbol{\gamma}\right)\right) \boldsymbol{I}+{ }^{2} \kappa\left(\operatorname{tr}\left({ }^{(2)} \gamma\right)\right) \boldsymbol{I} \\
{ }^{m} \boldsymbol{Q}_{s}=2\left({ }^{m} \eta\right)\left({ }^{t} \Theta \boldsymbol{J}\right)+{ }^{m} \kappa\left(\operatorname{tr}\left({ }^{t} \Theta \boldsymbol{J}\right)\right) \boldsymbol{I} \\
{ }^{m} \boldsymbol{Q}_{a}=2\left({ }^{a} m \eta_{1}\right)\left({ }_{i}^{t \Theta} \boldsymbol{J}\right)
\end{gathered}
$$

We can write (9.8)-(9.10) as follows

$$
\begin{gathered}
{ }_{d}\left({ }_{s}^{(0)} \overline{\boldsymbol{\sigma}}\right)+\lambda\left({ }_{d}\left({ }_{s}^{(1)} \overline{\boldsymbol{\sigma}}\right)\right)={ }^{\sigma} \boldsymbol{Q}_{s} \\
{ }_{s}^{(0)} \overline{\boldsymbol{m}}+{ }^{m} \lambda\left({ }_{s}^{(1)} \overline{\boldsymbol{m}}\right)={ }^{m} \boldsymbol{Q}_{s} \\
{ }_{a}^{(0)} \overline{\boldsymbol{m}}+{ }^{a} \lambda\left({ }_{a}^{(1)} \overline{\boldsymbol{m}}\right)={ }^{m} \boldsymbol{Q}_{a}
\end{gathered}
$$

Equations (10.4)-(10.6) are first order differential equations in time in $\left.{ }_{d}^{(0)} \bar{s}\right),{ }_{s}^{(0)} \overline{\boldsymbol{m}}$ and ${ }_{a}^{(0)} \overline{\boldsymbol{m}}$, hence can be integrated using the following: The differential equation

$$
\frac{\mathrm{d} \phi}{\mathrm{d} x}+P(x) \phi=Q(x)
$$

has the solution

$$
\phi=\mathrm{e}^{-\int P(x) \mathrm{d} x}\left[\int Q(x) \mathrm{e}^{\int P(x) \mathrm{d} x} \mathrm{~d} x+C\right]
$$

where $C$ is a constant of integration. We consider (10.4) and rewrite

$$
\left.{ }_{d}^{\left({ }^{(1)}\right.} \overline{\boldsymbol{\sigma}}\right)+\frac{1}{\lambda}{ }_{d}\left({ }_{s}^{(0)} \overline{\boldsymbol{\sigma}}\right)=\frac{1}{\lambda}{ }^{\sigma} \boldsymbol{Q}_{s}
$$

Hence using (10.7) and (10.8) we can write

$$
\begin{aligned}
{ }_{d}\left({ }_{s}^{(0)} \overline{\boldsymbol{\sigma}}\right) & =\mathrm{e}^{-\int 1 \lambda \mathrm{d} t}\left[\int \frac{1}{\lambda}\left({ }^{\sigma} \boldsymbol{Q}_{s}\right) \mathrm{e}^{j 1 \lambda \mathrm{d} t} \mathrm{~d} t+\boldsymbol{C}\right] \\
& =\mathrm{e}^{-t \lambda}\left[\int \frac{1}{\lambda}\left({ }^{\sigma} \boldsymbol{Q}_{s}\right) \mathrm{e}^{t^{\prime} \lambda} \mathrm{d} t^{\prime}+\boldsymbol{C}\right] \\
& =\frac{\int_{-\infty}^{t} \frac{1}{\lambda}\left({ }^{\sigma} \boldsymbol{Q}_{s}\left(t^{\prime}\right)\right) \mathrm{e}^{t^{\prime} \lambda} \mathrm{d} t^{\prime}}{\mathrm{e}^{t \lambda}}+\boldsymbol{C} \mathrm{e}^{-t \lambda}
\end{aligned}
$$

Based on reference [46] choice of $-\infty$ is arbitrary. Some other value could result in different value of $\boldsymbol{C}$. If we prescribe that the stress in the fluid is finite at $t=-\infty$, we must choose $\boldsymbol{C}$ to be zero. We must also check the first term in (10.10), since both numerator and denominator go to zero as $t$ goes to $-\infty$. Using L'Hôpital's rule we get: 


$$
\lim _{t \rightarrow-\infty d}\left({ }^{(0)} \bar{\sigma}\right)=\lim _{t \rightarrow-\infty} \frac{\frac{1}{\lambda}\left({ }^{\sigma} \boldsymbol{Q}_{s}(t)\right) \mathrm{e}^{t \lambda}}{\frac{1}{\lambda} \mathrm{e}^{t \lambda}}={ }^{\sigma} \boldsymbol{Q}_{s}(-\infty)
$$

Thus, if ${ }^{\sigma} \boldsymbol{Q}_{s}(-\infty)$ is finite, the stress is finite at $t=-\infty$, hence (10.10) reduces to

$$
{ }_{d}\left({ }_{s}^{(0)} \overline{\boldsymbol{\sigma}}\right)=\int_{-\infty}^{t}\left(\frac{1}{\lambda} \mathrm{e}^{-\left(t-t^{\prime}\right) \lambda}\right) \sigma \boldsymbol{Q}_{s}\left(t^{\prime}\right) \mathrm{d} t^{\prime}
$$

The quantity in the bracket in the integrand in (10.12) is called "retardation modulus" for ${ }_{d}\left({ }_{s}^{(0)} \bar{\sigma}\right)$. When ${ }^{\sigma} \boldsymbol{Q}_{s}$ only contains $2 \eta\left({ }^{(1)} \gamma\right)$ term, we can obtain relaxation modulus for ${ }_{d}\left(\begin{array}{c}{ }^{(0)} \\ s\end{array} \bar{\sigma}\right)$ from (10.12). This is straight forward. We omit this here as it requires approximating ${ }^{\sigma} \boldsymbol{Q}_{s}$. Retardation modulus is as good a measure of rheology as relaxation modulus. Using similar approach we can also derive the following from (10.5) and (10.6), first by rewriting them by dividing by ${ }^{m} \lambda$ and ${ }^{a m} \lambda$ respectively.

$$
\begin{aligned}
& { }_{s}^{(1)} \overline{\boldsymbol{m}}+\left(\frac{1}{{ }^{m} \lambda}\right){ }_{s}^{(0)} \overline{\boldsymbol{m}}=\left(\frac{1}{{ }^{m} \lambda}\right)\left({ }^{m} \boldsymbol{Q}_{s}\right) \\
& \text { and } \quad{ }_{a}^{(1)} \overline{\boldsymbol{m}}+\left(\frac{1}{{ }^{a}{ }^{m} \lambda}\right){ }_{a}^{(0)} \overline{\boldsymbol{m}}=\left(\frac{1}{{ }^{a}{ }^{m} \lambda}\right)\left({ }^{m} \boldsymbol{Q}_{a}\right)
\end{aligned}
$$

and then following the derivation for ${ }_{d}\left(\begin{array}{c}(0) \\ s\end{array} \bar{\sigma}\right)$

$$
\begin{aligned}
& { }_{s}^{(0)} \overline{\boldsymbol{m}}=\int_{-\infty}^{t}\left(\frac{1}{{ }^{m} \lambda} \mathrm{e}^{-\left(t-t^{\prime}\right)^{s^{m}} \lambda}\right){ }^{m} \boldsymbol{Q}_{s}\left(t^{\prime}\right) \mathrm{d} t^{\prime} \\
& { }_{a}^{(0)} \overline{\boldsymbol{m}}=\int_{-\infty}^{t}\left(\frac{1}{{ }^{a} \lambda} \mathrm{e}^{-\left(t-t^{\prime}\right)^{a^{m}} \lambda}\right){ }^{m} \boldsymbol{Q}_{a}\left(t^{\prime}\right) \mathrm{d} t^{\prime}
\end{aligned}
$$

The terms in the brackets in (10.15) and (10.16) are called retardation modulus for ${ }_{s}^{(0)} \overline{\boldsymbol{m}}$ and ${ }_{a}^{(0)} \overline{\boldsymbol{m}}$, respectively.

\section{Remarks}

1) We observe that the non-classical polymeric fluids have relaxation mechanism due to ${ }_{d}\left({ }_{s}^{(0)} \overline{\boldsymbol{\sigma}}\right)$ as well as ${ }_{s}^{(0)} \overline{\boldsymbol{m}}$ and ${ }_{a}^{(0)} \overline{\boldsymbol{m}}$ when balance of moments of moments is not considered as a balance law.

2) When the balance of moments of moments is used as a balance law ${ }^{(0)} \overline{\boldsymbol{m}}$ is symmetric, hence ${ }_{s}^{(0)} \overline{\boldsymbol{m}}={ }^{(0)} \overline{\boldsymbol{m}}$ and ${ }_{a}^{(0)} \overline{\boldsymbol{m}}=0$. Thus in this case the relaxation mechanism is only due to ${ }_{d}\left({ }_{s}^{(0)} \overline{\boldsymbol{\sigma}}\right)$ and ${ }_{s}^{(0)} \overline{\boldsymbol{m}}$.

\section{Summary and Conclusions}

This paper considers conservation and balance laws for non-classical continuum theory for fluent continua to present derivations of the constitutive theories for thermoviscoelastic fluids, both compressible and incompressible. The non-classical continuum theory and the corresponding constitutive theories incorporate symmetric as well as antisymmetric parts of the velocity gradient tensor. The rotation rates defined by the antisymmetric part of the velocity gradient tensor 
(referred to as internal rotation rates) act about the axes of a triad located at each material point (or a location). The axes of the triad are parallel to the axes of the fixed $x$-frame. The constitutive variables are determined from the entropy inequality as well as other conservation and balance laws. The argument tensors of the constitutive variables are decided using conjugate pairs in the entropy inequality as well as by considering other desired physics that is not obvious from the entropy inequality. The constitutive theories are derived using representation theorem. All constitutive theories are based on integrity. Simplified linear, quasilinear, and nonlinear forms of the constitutive theories are presented and compared with parallel constitutive theories for such fluent continua in classical continuum theories. In the following we present a summary of the significant aspects of the work presented in this paper.

1) The constitutive theories for stress and moment tensors are ordered rate constitutive theories up to any desired orders. That is, the constitutive theories utilize convected time derivatives of the constitutive variables (up to any desired orders) as well as convected time derivatives of their argument tensors (also up to any desired orders).

2) All constitutive theories are based on integrity, hence utilize complete basis. The only assumption is in the Taylor series expansion of the coefficients in the linear combination (truncated after linear terms) about a known configuration. These theories provide more comprehensive description of the constitution of the deforming matter.

3) The derivations of the constitutive theories are basis independent due to basis independent choice of the constitutive variables as well as their argument tensors. By appropriate choices of the bases for the constitutive variables and their argument tensors, the constitutive theories can be easily made basis specific. For example, if $\left.{ }_{d}^{\left({ }_{s}^{(0)}\right.} \overline{\boldsymbol{\sigma}}\right),{ }_{s}^{(0)} \overline{\boldsymbol{m}}$ and ${ }_{a}^{(0)} \overline{\boldsymbol{m}}$ are chosen as contravariant measures $\left({ }_{d}\left({ }_{s} \overline{\boldsymbol{\sigma}}^{(0)}\right),{ }_{s} \overline{\boldsymbol{m}}^{(0)}{ }^{d}\right.$ and $\left.{ }_{a} \overline{\boldsymbol{m}}^{(0)}\right)$, then their conjugates must be in covariant basis and vice-versa.

4) In the non-classical thermoviscoelastic fluids considered in this paper, the mechanisms of energy storage, dissipation of mechanical work, and rheology are due to $\left.{ }_{d}^{\left({ }_{s}^{(0)}\right.} \overline{\boldsymbol{\sigma}}\right),{ }_{s}^{(0)} \overline{\boldsymbol{m}}$ and ${ }_{a}^{(0)} \overline{\boldsymbol{m}}$, whereas in the case of classical thermoviscoelastic fluids, these mechanisms are only due to ${ }_{d}\left({ }_{s}^{(0)} \bar{\sigma}\right)$.

5) It has been shown by Yang et al. [75] and Surana et al. [76] [77] that the balance of moment of moments is a necessary balance law in non-classical continuum theories to ensure that the deforming volume of matter is in equilibrium. In the presence of this balance law, the Cauchy moment tensor becomes symmetric. In this paper we have presented derivations of the constitutive theories when the balance of moment of moments is not a balance law. This is a more general case. When the balance of moment of moments is a balance law, the Cauchy moment tensor becomes symmetric, i.e., ${ }^{(0)} \overline{\boldsymbol{m}}={ }_{s}^{(0)} \overline{\boldsymbol{m}}$ and ${ }_{a}^{(0)} \overline{\boldsymbol{m}}=0$.

6) Retardation moduli are derived for ${ }_{d}\left({ }_{s}^{(0)} \overline{\boldsymbol{\sigma}}\right),{ }_{s}^{(0)} \overline{\boldsymbol{m}}$ and ${ }_{a}^{(0)} \overline{\boldsymbol{m}}$. It can be shown that with some assumptions relaxation moduli can be derived from these. 
When comparing with classical continuum theories for polymers, we find additional mechanisms of dissipation and rheology in the non-classical thermoviscoelastic fluids.

7) As shown in this paper, the constitutive theories based on integrity are almost always nonlinear in their argument tensors. Their linearizations are perfectly valid if limited physics is of interest, however the conclusions that may be drawn from the superposition of linear constitutive theories are obviously invalid for the constitutive theories based on integrity. An example would be linear constitutive theories for ${ }_{a}^{(0)} \overline{\boldsymbol{m}}$ and ${ }_{a}^{(0)} \overline{\boldsymbol{m}}$, suggesting a constitutive theory for $\left({ }_{s}^{(0)} \overline{\boldsymbol{m}}+{ }_{a}^{(0)} \overline{\boldsymbol{m}}\right)$, a non-symmetric tensor in terms of non-symmetric argument tensors is obviously invalid.

8) Simplified form of the rate constitutive theories are derived to show that currently used Maxwell, Oldroyd-B, and Giesekus constitutive theories in classical continuum mechanics are in fact a subset of the more general non-classical theories presented in this paper.

In conclusion the work presented in this paper utilizes a consistent thermodynamic framework for non-classical fluent continua and presents derivations of constitutive theories for thermoviscoelastic fluids with memory, compressible and incompressible, by incorporating internal rotation rates due to the velocity gradient tensor at a material point. The paper contains thermodynamically consistent derivations of constitutive theories in which all possible mechanisms of energy storage, dissipation, and rheology are considered. Memory (or rheology) mechanism is incorporated by considering rate constitutive theories in terms of the rates of constitutive variables.

\section{Acknowledgements}

The first and third authors are grateful for the support provided by their endowed professorships during the course of this research. The second author is grateful for the financial support provided by the department of mechanical engineering of the University of Kansas, Lawrence, KS.

\section{Conflicts of Interest}

The authors declare no conflicts of interest regarding the publication of this paper.

\section{References}

[1] Surana, K.S. Powell, M.J. and Reddy, J.N. (2015) A More Complete Thermodynamic Framework for Solid Continua. Journal of Thermal Engineering, 1, 1-13. https://doi.org/10.18186/jte.17430

[2] Surana, K.S., Reddy, J.N., Nunez, D. and Powell, M.J. (2015) A Polar Continuum Theory for Solid Continua. International Journal of Engineering Research and Industrial Applications, 8, 77-106.

[3] Surana, K.S., Powell, M.J. and Reddy, J.N. (2015) A More Complete Thermodynamic Framework for Fluent Continua. Journal of Thermal Engineering, 1, 14-30. 
[4] Surana, K.S., Powell, M.J. and Reddy, J.N. (2015) A Polar Continuum Theory for Fluent Continua. International Journal of Engineering Research and Industrial Applications, 8, 107-146.

[5] Voigt, W. (1887) Theoretische Studien über die Wissenschaften zu Elastizitätsverhältnisse der Krystalle. Abhandl. Ges. Göttingen, 34.

[6] Voigt, W. (1894) über Medien ohne innere Kräfte und eine durch sie gelieferte mechanische Deutung der Maxwell-Hertzschen Gleichungen. Göttingen Abhandl, 72-79.

[7] Cosserat, E. and Cosserat, F. (1909) Théorie des corps déformables. Hermann, Paris.

[8] W. Günther. (1958) Zur Statik und Kinematik des Cosseratschen Kontinuums. $A b$ handl. Braunschweig. Wiss. Ges, 10, 195-213.

[9] Grioli, G. (1960) Elasticità Asimmetrica. Annali di Matematica Pura ed Applicata, 50, 389-417. https://doi.org/10.1007/BF02414525

[10] Aero, E.L. and Kuvshinskii, E.V. (1961) Fundamental Equations of the Theory of Elastic Media with Rotationally Interacting Particles. Soviet Physics, Solid State, 2, 1272-1281.

[11] Schäfer, H. (1962) Versuch einer Elastizitätstheorie des Zweidimensionalen Ebenen Cosserat-Kontinuums. Miszellaneen der Angewandten Mechanik, 277-292.

[12] Truesdell, C.A. and Toupin, R.A. (1960) The Classical Field Theories of Mechanics. In: Flügge, S., Ed., Handbuch der Physik, Volume 3, Springer-Verlag, Berlin.

[13] Mindlin, R.D. and Tiersten, H.F. (1962) Effects of Couple-Stresses in Linear Elasticity. Archive for Rational Mechanics and Analysis, 11, 415-448. https://doi.org/10.1007/BF00253946

[14] Toupin, R.A. (1962) Elastic Materials with Couple-Stresses. Archive for Rational Mechanics and Analysis, 11, 385-414. https://doi.org/10.1007/BF00253945

[15] Koiter, W.T. (1964) Couple Stresses in the Theory of Elasticity, I and II. Proceedings Series B, Koninklijke Nederlandse Akademie van Wetenschappen, 67, 17-44.

[16] Eringen, A.C. (1962) Nonlinear Theory of Continuous Media. McGraw-Hill, New York.

[17] Eringen, A.C. and Suhubi, E.S. (1964) Nonlinear Theory of Simple Micro-Elastic Solids I. International Journal of Engineering Science, 2, 189-203. https://doi.org/10.1016/0020-7225(64)90004-7

[18] Eringen, A.C. and Suhubi, E.S. (1964) Nonlinear Theory of Simple Micro-Elastic Solids II. International Journal of Engineering Science, 2, 389-404. https://doi.org/10.1016/0020-7225(64)90004-7

[19] Eringen, A.C. (1964) Simple Microuids. International Journal of Engineering Science, 2, 205-217. https://doi.org/10.1016/0020-7225(64)90005-9

[20] Eringen, A.C. (1964) Mechanics of Micromorphic Materials. In: Gortler, H., Ed., Proceeding of 11 th International Congress of Applied Mechanics, Springer-Verlag, Berlin, 131-138.

[21] Mindlin, R.D. (1964) Micro-Structure in Linear Elasticity. Archive for Rational Mechanics and Analysis, 16, 51-78. https://doi.org/10.1007/BF00248490

[22] Green, A.E. and Rivlin, R.S. (1964) Multipolar Continuum Mechanics. Archive for Rational Mechanics and Analysis, 17, 113-147. https://doi.org/10.1007/BF00253051

[23] Mindlin, R.D. (1965) Stress Functions for a Cosserat Continuum. International Journal of Solids and Structures, 1, 265-271. https://doi.org/10.1016/0020-7683(65)90033-8 
[24] Brand, M. and Rubin, M.B. (2007) A Constrained Theory of a Cosserat Point for the Numerical Solution of Dynamic Problems of Non-Linear Elastic Rods with Rigid Cross-Sections. International Journal of Non-Linear Mechanics, 42, 216-232. https://doi.org/10.1016/j.ijnonlinmec.2006.10.002

[25] Cao, D.Q. and Tucker, R.W. (2008) Nonlinear Dynamics of Elastic Rods Using the Cosserat Theory: Modelling and Simulation. International Journal of Solids and Structures, 45, 460-477. https://doi.org/10.1016/j.ijsolstr.2007.08.016

[26] Steinmann, P. (1994) A Micropolar Theory of Finite Deformation and Finite Rotation Multiplicative Elastoplasticity. International Journal of Solids and Structures, 31, 1063-1084. https://doi.org/10.1016/0020-7683(94)90164-3

[27] Srinivasa, A.R. and Reddy, J.N. (2013) A Model for a Constrained, Finitely Deforming, Elastic Solid with Rotation Gradient Dependent Strain Energy, and Its Specialization to von Kármán Plates and Beams. Journal of Mechanics and Physics of Solids, 61, 873-885. https://doi.org/10.1016/j.jmps.2012.10.008

[28] Segerstad, P.H., Toll, S. and Larsson, R. (2008) A Micropolar Theory for the Finite Elasticity of Open-Cell Cellular Solids. Proceedings of the Royal Society A, 465, 843-865. https://doi.org/10.1098/rspa.2008.0267

[29] Eringen, A.C. (1967) Mechanics of Continua. John Wiley and Sons, Hoboken.

[30] Eringen, A.C. (1968) Mechanics of Micromorphic Continua. In: Kroner, E., Ed., Mechanics of Generalized Continua, Springer, Berlin, 18-35.

https://doi.org/10.1007/978-3-662-30257-6_2

[31] Eringen, A.C. (1966) A Unified Theory of Thermomechanical Materials. International Journal of Engineering Science, 4, 179-202. https://doi.org/10.1016/0020-7225(66)90022-X

[32] Eringen, A.C. (1967) Linear Theory of Micropolar Viscoelasticity. International Journal of Engineering Science, 5, 191-204. https://doi.org/10.1016/0020-7225(67)90004-3

[33] Eringen, A.C. (1968) Theory of Micropolar Elasticity. In: Liebowitz, H., Ed., Fracture, Academic Press, New York, 621-729.

[34] Eringen, A.C. (1970) Balance Laws of Micromorphic Mechanics. International Journal of Engineering Science, 8, 819-828. https://doi.org/10.1016/0020-7225(70)90084-4

[35] Eringen, A.C. (1972) Theory of Micromorphic Materials with Memory. International Journal of Engineering Science, 10, 623-641. https://doi.org/10.1016/0020-7225(72)90089-4

[36] Eringen, A.C. (1990) Theory of Thermo-Microstretch Fluids and Bubbly Liquids. International Journal of Engineering Science, 28, 133-143. https://doi.org/10.1016/0020-7225(90)90063-O

[37] Eringen, A.C. (1999) Microcontinuum Field Theories. Springer, New York. https://doi.org/10.1007/978-1-4612-0555-5

[38] Surana, K.S. (2015) Advanced Mechanics of Continua. CRC/Taylor and Francis, Boca Raton.

[39] Surana, K.S., Nunez, D., Reddy, J.N. and Romkes, A. (2013) Rate Constitutive Theory for Ordered Thermofluids. Journal of Continuum Mechanics and Thermodynamics, 25, 626-662. https://doi.org/10.1007/s00161-012-0257-6

[40] Surana, K.S., Nunez, D., Reddy, J.N. and Romkes, A. (2014) Rate Constitutive Theory for Ordered Thermoviscoelastic Fluids: Polymers. Continuum Mechanics and Thermodynamics, 26, 143-181. https://doi.org/10.1007/s00161-013-0295-8 
[41] Surana, K.S., Nunez, D. and Giesekus, R.J.N. (2013) Constitutive Model for Thermoviscoelastic Fluids Based on Ordered Rate Constitutive Theories. Journal of Research Updates in Polymer Science, 2, 232-260.

[42] Surana, K.S., Nunez, D., Reddy, J.N. and Romkes, A. (2012) Rate Constitutive Theory for Ordered Thermoelastic Solids. Annals of Solid and Structural Mechanics, 3, 27-54. https://doi.org/10.1007/s12356-012-0027-y

[43] Surana, K.S., Moody, T.C. and Reddy, J.N. (2014) Rate Constitutive Theories of Order Zero in Lagrangian Description for Thermoelastic Solids. Mechanics of Advanced Materials and Structures, 22, 440-450. https://doi.org/10.1080/15376494.2013.778617

[44] Surana, K.S., Moody, T.C. and Reddy, J.N. (2013) Ordered Rate Constitutive Theories in Lagrangian Description for Thermoviscoelastic Solids without Memory. Acta Mechanica, 224, 2785-2816. https://doi.org/10.1007/s00707-013-0893-3

[45] Surana, K.S., Moody, T.C. and Reddy, J.N. (2014) Ordered Rate Constitutive Theories in Lagrangian Description for Thermoviscoelastic Solids with Memory. Acta Mechanica, 226, 157-178. https://doi.org/10.1007/s00707-014-1173-6

[46] Bird, R.B., Armstrong, R.C. and Hassager, O. (1987) Dynamics of Polymeric Liquids, Volume 1, Fluid Mechanics. 2nd Edition, John Wiley and Sons, Hoboken.

[47] Surana, K.S., Powell, M.J. and Reddy, J.N. (2015) Constitutive Theories for Internal Polar Thermoelastic Solid Continua. Journal of Pure and Applied Mathematics. Advances and Applications, 14, 89-150.

[48] Surana, K.S., Powell, M.J. and Reddy, J.N. (2015) Ordered Rate Constitutive Theories for Internal Polar Thermouids. International Journal of Mathematics, Science, and Engineering Applications, 9, 51-116.

[49] Surana, K.S., Joy, A.D. and Reddy, J.N. (2016) A Non-Classical Internal Polar Continuum Theory for Finite Deformation and Finite Strain in Solids. International Journal of Pure and Engineering Mathematics, 4, 59-97.

[50] Surana, K.S., Joy, A.D. and Reddy, J.N. (2016) A Non-Classical Internal Polar Continuum Theory for Finite Deformation of Solids Using First Piola-Kirchhoff Stress Tensor. Journal of Pure and Applied Mathematics. Advances and Applications, 16, $1-41$.

[51] Surana, K.S., Joy, A.D. and Reddy, J.N. (2017) A Non-Classical Continuum Theory for Solids Incorporating Internal Rotations and Rotations of Cosserat Theories. Continuum Mechanics and Thermodynamics, 29, 665-698. https://doi.org/10.1007/s00161-017-0554-1

[52] Surana, K.S., Joy, A.D. and Reddy, J.N. (2017) A Non-Classical Continuum Theory for Fluids Incorporating Internal and Cosserat Rotation Rates. Continuum $\mathrm{Me}$ chanics and Thermodynamics, 29, 1249-1289. https://doi.org/10.1007/s00161-017-0579-5

[53] Surana, K.S., Joy, A.D. and Reddy, J.N. (2018) Ordered Rate Constitutive Theories for Thermoviscoelastic Solids without Memory Incorporating Internal and Cosserat Rotations. Acta Mechanica, 229, 3189-3213. https://doi.org/10.1007/s00707-018-2163-x

[54] Reiner, M. (1945) A Mathematical Theory of Dilatancy. American Journal of Mathematics, 67, 350-362. https://doi.org/10.2307/2371950

[55] Todd, J.A. (1948) Ternary Quadratic Types. Philosophical Transactions of the Royal Society of London. Series A: Mathematical and Physical Sciences, 241, 399-456. https://doi.org/10.1098/rsta.1948.0025 
[56] Rivlin, R.S. and Ericksen, J.L. (1955) Stress-Deformation Relations for Isotropic Materials. Journal of Rational Mechanics and Analysis, 4, 323-425. https://doi.org/10.1512/iumj.1955.4.54011

[57] Rivlin, R.S. (1955) Further Remarks on the Stress-Deformation Relations for Isotropic Materials. Journal of Rational Mechanics and Analysis, 4, 681-702.

[58] Wang, C.C. (1969) On Representations for Isotropic Functions, Part I. Archive for Rational Mechanics and Analysis, 33, 249-267. https://doi.org/10.1007/BF00281278

[59] Wang, C.C. (1969) On Representations for Isotropic Functions, Part II. Archive for Rational Mechanics and Analysis, 33, 268-287. https://doi.org/10.1007/BF00281279

[60] Wang, C.C. (1970) A New Representation Theorem for Isotropic Functions, Part I and Part II. Archive for Rational Mechanics and Analysis, 36, 166-223. https://doi.org/10.1007/BF00272241

[61] Wang, C.C. (1971) Corrigendum to Representations for Isotropic Functions. Archive for Rational Mechanics and Analysis, 43, 392-395. https://doi.org/10.1007/BF00252004

[62] Smith, G.F. (1970) On a Fundamental Error in Two Papers of C.C. Wang, on Representations for Isotropic Functions, Part I and Part II. Archive for Rational Mechanics and Analysis, 36, 161-165. https://doi.org/10.1007/BF00272240

[63] Smith, G.F. (1971) On Isotropic Functions of Symmetric Tensors, Skew-Symmetric Tensors and Vectors. International Journal of Engineering Science, 9, 899-916. https://doi.org/10.1016/0020-7225(71)90023-1

[64] Spencer, A.J.M. and Rivlin, R.S. (1959) The Theory of Matrix Polynomials and Its Application to the Mechanics of Isotropic Continua. Archive for Rational Mechanics and Analysis, 2, 309-336. https://doi.org/10.1007/BF00277933

[65] Spencer, A.J.M. and Rivlin, R.S. (1960) Further Results in the Theory of Matrix Polynomials. Archive for Rational Mechanics and Analysis, 4, 214-230. https://doi.org/10.1007/BF00281388

[66] Spencer, A.J.M. (1971) Theory of Invariants. Chapter 3 Treatise on Continuum Physics I. Academic Press, Cambridge. https://doi.org/10.1016/B978-0-12-240801-4.50008-X

[67] Boehler, J.P. (1977) On Irreducible Representations for Isotropic Scalar Functions. Journal of Applied Mathematicsand Mechanics, 57, 323-327. https://doi.org/10.1002/zamm.19770570608

[68] Zheng, Q.S. (1993) On the Representations for Isotropic Vector-Valued, Symmetric Tensor-Valued and Skew-Symmetric Tensor-Valued Functions. International Journal of Engineering Science, 31, 1013-1024. https://doi.org/10.1016/0020-7225(93)90109-8

[69] Zheng, Q.S. (1993) On Transversely Isotropic, Orthotropic and Relatively Isotropic Functions of Symmetric Tensors, Skew-Symmetric Tensors, and Vectors. International Journal of Engineering Science, 31, 1399-1453. https://doi.org/10.1016/0020-7225(93)90005-F

[70] Hill, R. (1968) On Constitutive Inequalities for Simple Materials I. Journal of the Mechanics and Physics of Solids, 16, 229-242. https://doi.org/10.1016/0022-5096(68)90031-8

[71] Hill, R. (1968) On Constitutive Inequalities for Simple Materials II. Journal of the Mechanics and Physics of Solids, 16, 315-322. https://doi.org/10.1016/0022-5096(68)90018-5

[72] Hill, R. (1970) Constitutive Inequalities for Isotropic Elastic Solids under Finite 
Strain. Proceedings of the Royal Society of London. Series A, 314, 457-472. https://doi.org/10.1098/rspa.1970.0018

[73] Hill, R. (1978) Aspects of Invariance in Solid Mechanics. Advances in Applied Mechanics, 18, 1-72.

[74] Murnaghan, F.D. (1951) Finite Deformation of an Elastic Solid. John Wiley \& Sons, New York.

[75] Yang, F., Chong, A.C.M., Lam, D.C.C. and Tong, P. (2002) Couple Stress Based Strain Gradient Theory for Elasticity. International Journal of Solids and Structures, 39, 2731-2743. https://doi.org/10.1016/S0020-7683(02)00152-X

[76] Surana, K.S. Shanbhag, R.S. and Reddy, J.N. (2018) Necessity of Balance of Moment of Moments Balance Law in Non-Classical Continuum Theories for Solid Continua. Meccanica, 53, 2939-2972. https://doi.org/10.1007/s11012-018-0851-1

[77] Surana, K.S., Long, S.W. and Reddy, J.N. (2018) Necessity of Balance of Moment of Moments Balance Law in Non-Classical Continuum Theories for Fluent Continua. Acta Mechanica, 229, 2801-2833. https://doi.org/10.1007/s00707-018-2143-1 